\title{
Sulfonic Acid Libraries Attained Through Opening of 2-Sulfobenzoic Acid Anhydride
}

\author{
Richard I. Robinson, ${ }^{\text {[a] }}$ John C. Stephens, ${ }^{\text {[a] }}$ Steve M. Worden, ${ }^{\text {[a] }}$ Alexander J. Blake, ${ }^{\text {[a] }}$ \\ Claire Wilson, ${ }^{\text {[a] }}$ and Simon Woodward ${ }^{\star[a]}$
}

Keywords: Molecular diversity / Amino alcohols / Sulfonic acids / S ligands / N,O ligands / Hydrogen bonding

2-Sulfobenzoic acid anhydride opens cleanly with $N$-Bocprotected $\alpha$-amino alcohols to afford zwitterionic esters 1,2$\mathrm{C}_{6} \mathrm{H}_{4}\left(\mathrm{SO}_{3}{ }^{-}\right)\left(\mathrm{CO}_{2} \mathrm{CH}_{2} \mathrm{CHR}^{1} \mathrm{NH}_{3}{ }^{+}\right)\left(\mathrm{R}^{1}=\mathrm{H}\right.$, alkyl), the species $\mathrm{R}^{1}=i \mathrm{Pr}$ is crystallographically characterised. Dehydration of these species affords zwitterionic oxazoline sulfonic acid derivatives $1,2-\mathrm{C}_{6} \mathrm{H}_{4}\left(\mathrm{SO}_{3}{ }^{-}\right)\left(\mathrm{C}^{\mathrm{A}}=\mathrm{NH}^{+} \mathrm{CHR}^{1} \mathrm{CH}_{2} \mathrm{O}^{\mathrm{A}}\right)\left(\mathrm{C}^{\mathrm{A}}\right.$ and $\mathrm{O}^{\mathrm{A}}$ are bonded $\mathrm{C}-\mathrm{O}$ ) for $\mathrm{R}^{1}=i \operatorname{Pr}\left(\mathrm{X}\right.$-ray), $i \mathrm{Bu}, t \mathrm{Bu}, \mathrm{CH}_{2} \mathrm{Ph}$. Reaction with water regenerates the zwitterionic esters while exposure to $\mathrm{M}(\mathrm{OAc})_{2}(\mathrm{M}=\mathrm{Cu}, \mathrm{Pd})$ leads to the formation of the crystallographically characterised complexes $\mathrm{M}^{\mathrm{II}} \mathrm{L}_{2}(\mathrm{~L}=$ anion of the iPr-oxazolinesulfonic acid). Heating either the zwitterionic esters or oxazolines with $\mathrm{R}^{2} \mathrm{NH}_{2}\left[\mathrm{R}^{2}=\mathrm{CH}_{2} \mathrm{Ph}\right.$, $\mathrm{CH}_{2}\left(1-\mathrm{C}_{10} \mathrm{H}_{7}\right), R-\mathrm{CH}(\mathrm{Me}) \mathrm{Ph}$ ] leads to $\mathrm{S}_{\mathrm{N}} 2$ attack at the oxazoline methylene group leading to the amido sulfonic acids 1,2- $\mathrm{C}_{6} \mathrm{H}_{4}\left(\mathrm{CONHCHRCH} \mathrm{NHR}^{2}\right)\left(\mathrm{SO}_{3} \mathrm{H}\right)$ based on crystallographic studies on $\mathrm{R}^{1}=i \mathrm{Pr}, \mathrm{R}^{2}=\mathrm{CH}_{2}\left(1-\mathrm{C}_{10} \mathrm{H}_{7}\right)$.

(ㄷ Wiley-VCH Verlag GmbH \& Co. KGaA, 69451 Weinheim, Germany, 2004)

\section{Introduction}

One major theme of early 21 st century synthesis has become the preparation, through simple chemistry, of compound libraries. Such libraries find application in drug screening, catalysis and materials chemistry. ${ }^{[1]}$ Sulfonic acids, and their derived sulfonamides, are interesting compound classes to target in this arena as both potent pharmaceutical and catalytic ligand lead structures abound. ${ }^{[2,3]}$ We envisaged that the interesting sulfonic acid oxazolines $\mathbf{1}$ could be attained from either the esters $\mathbf{2}$ or the amides $\mathbf{3}$, and potentially functionalised further to sulfonamides. Either $\mathbf{2}$ or $\mathbf{3}$ should be available from suitable "click-reaction" ${ }^{[4]}$ opening of commercial 2-sulfobenzoic acid anhydride (4) with amino alcohols or suitable derivatives (Scheme 1). Under appropriate conditions it should be possible to attain a one-step synthesis. While this chemistry is unprecedented there are limited suggestions in patent, and related, literature that the equivalent transformations with diamines have been attained. ${ }^{[5]}$

\section{Results and Discussion}

\section{Oxazoline Synthesis}

Initial investigations were carried out using valinol as a representative $\alpha$-amino alcohol. Reaction with the anhy-

[a] School of Chemistry, The University of Nottingham, University Park, Nottingham, United Kingdom Fax: (internat.) +44-115-9513564 E-mail: simon.woodward@nottingham.ac.uk

- Supporting information for this article is available on the WWW under http://www.eurjoc.org or from the author.<smiles>[R]C1COC(c2ccccc2S(=O)(=O)O[Tl])=N1</smiles><smiles>[R]C([NH3+])COC(=O)c1ccccc1[S+](=O)[O-]</smiles><smiles>[R]C(CO)NC(=O)c1ccccc1S(=O)(=O)O</smiles>

2 or<smiles>C=CC</smiles><smiles>O=c1oc2ccccc2o1</smiles><smiles>[R]C(N)CO</smiles>
(or derivative)

4

Scheme 1. Preparative routes to oxazoline sulfonic acids.

dride 4 under Dean-Stark conditions in $\mathrm{PhCl}$ pleasingly led to direct formation of an oxazoline formulated as tautomer 1a $(\mathrm{R}=i \mathrm{Pr})$ in $50-60 \%$ recrystallised yield after an overnight reflux. Purification of $\mathbf{1 a}$ is complicated by its polar nature and by the presence of two additional species. Proton NMR and IR spectroscopy confirmed these as residual ester 2a $\left(v_{\mathrm{CO}}=1736 \mathrm{~cm}^{-1}\right)$ and amide $\mathbf{3 a}\left(v_{\mathrm{CO}}=\right.$ $\left.1653 \mathrm{~cm}^{-1}\right)$. The sulfonic acid $1 \mathrm{a}$ could not be chromatographed but crystallised readily from the reaction residue using hot ethanol. Other derivatives of $\mathbf{1}(\mathrm{R}=$ alkyl) were not as crystalline and could not be separated from the ester/ 
amide mixtures. Even under prolonged heating complete conversion of any amino alcohol and $\mathbf{4}$ into $\mathbf{1}$ could not be attained, some $\mathbf{2}$ and $\mathbf{3}$ always remained (amoung other products). The origin of this observation was revealed by monitoring the reaction of valinol with 4 by ${ }^{1} \mathrm{H}$ NMR spectroscopy in $\mathrm{CDCl}_{3}$. At room temperature $\mathbf{2 a}$ and $\mathbf{3 a}$ are formed in a 1:1 ratio from 4 and valinol, although the reaction is not clean. We reasoned that the amine function is more nucleophilic and opens 4 quickly, but subsequent deprotonation of the resulting ammonium species by more valinol is rapid leading to an eqimolar mixture of ester $\mathbf{2 a}$ and amide 3a. Addition of valinol to 4 in the presence of $\mathrm{NEt}_{3}$ allowed greater formation of $\mathbf{3 a}$ (based on ${ }^{1} \mathrm{H}$ NMR spectra of the crude product) but it's high water solubility, and the presence of $\mathrm{NHEt}_{3} \mathrm{Cl}$, prevented its isolation. Importantly, heating the crude amide mixture containing $\mathbf{3 a}$ did not fashion any oxazoline indicating that it is the formation of 3 that limits the conversion of 4 . Reaction of $O$-TMS protected valinol with $\mathbf{4}$ was also attempted to provided a route to 1a (via 3a). However, subsequent heating, under DeanStark conditions in $\mathrm{PhCl}$, of the $O$-TMS analogue of $\mathbf{3 a}$ also afforded no oxazoline 1a. In contrast, we could readily confirm that the ester $\mathbf{2 a}(\mathrm{R}=i \mathrm{Pr})$ was cleanly converted to oxazoline 1a in quantitative yield under identical dehydrating conditions. These observations show that ester $\mathbf{2 a}$ is the key intermediate in the preparation of $\mathbf{1 a}$ and that a general approach to $\mathbf{1}$ should be attainable by such a route. To overcome the problems associated with co-formation of 3 in the reaction mixtures we utilised $N$-Boc-protected amino alcohols and realised excellent yields of $\mathbf{2}$ for a range of substituents (Scheme 2). The Boc group ensures clean formation of the desired ester and is subsequently spontaneously deprotected by the unmasked sulfonic acid, presumably via $\mathbf{5}$. Because of these features no added acid is required for the transformation. When anhydrous $\mathrm{MeCN}$ is used as the solvent at $60{ }^{\circ} \mathrm{C}, 2$ precipitates from the reaction mixture as a white solid, and this has been found to be analytically pure in all the cases we have tried. Reactions carrried out at $>5 \mathrm{~g}$ gave out large quantities of heat and gas, so precautionary steps should be employed regarding the rate of addition of the $N$-Boc-amino alcohol in such cases. Species $\mathbf{2}$ are extremely stable and were unchanged after storage for $>1$ year under standard laboratory conditions.

Dean-Stark dehydration of the esters 2 to 1 proceeds well in $\mathrm{PhCl}\left(135^{\circ} \mathrm{C}, 16 \mathrm{~h}\right)$, except in the cases where $\mathrm{R}=$ $\mathrm{H}, \mathrm{Me}$, where no product is isolated. This appears to be due solely to the insolubility of $\mathbf{2 e}-\mathbf{f}$ in the $\mathrm{PhCl}$ but attempts to ovecome this by changing the solvent were not successful. Lack of reactivity is not an issue as $\mathbf{2 e}-\mathbf{f}$ take part in other reactions, for example, $\mathbf{2 e}$ reacts with salicylaldehyde to provide the novel ligand $\mathbf{6}$ [Equation (1)]. The final oxazolines 1 are suseptible to slow hydrolysis on storage under non anhydrous conditions. Heating samples of $\mathbf{1}$ in water facilitates this degradation. For example, 1a is completely hydrolysed within $1 \mathrm{~h}$ in warm $\left(45-55^{\circ} \mathrm{C}\right) \mathrm{D}_{2} \mathrm{O}$. The formation of $\mathbf{2}$ by the hydrolysis of $\mathbf{1}$ is somewhat surprising, as amides are typically produced by acid-catalysed oxazoline
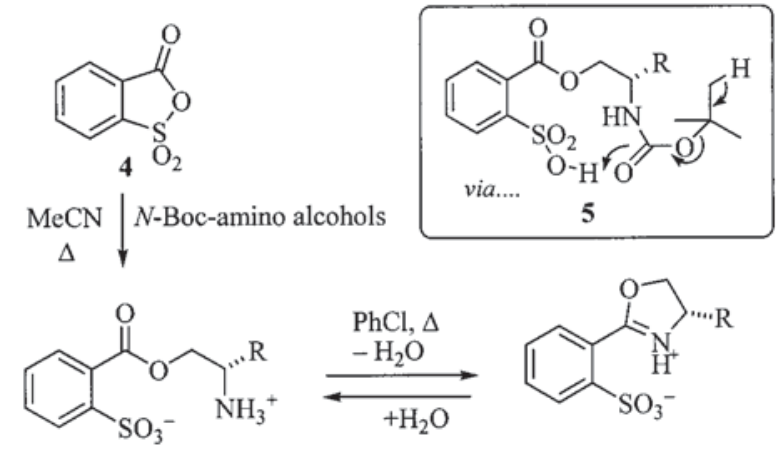

\begin{tabular}{l|c|l} 
2 & $\mathrm{R}$ & Yield/\% \\
\hline $\mathbf{a}$ & $i \mathrm{Pr}$ & 67 \\
$\mathbf{b}$ & $i \mathrm{Bu}$ & 68 \\
$\mathbf{c}$ & $t \mathrm{Bu}$ & 87 \\
$\mathbf{d}$ & $\mathrm{Bn}$ & 96 \\
$\mathbf{e}$ & $\mathrm{H}$ & 94 \\
$\mathbf{f}$ & $\mathrm{Me}$ & 60
\end{tabular}

\begin{tabular}{c|c|c}
$\mathbf{1}$ & $\mathrm{R}$ & Yield/\% \\
\hline $\mathbf{a}$ & $i \mathrm{Pr}$ & 100 \\
$\mathbf{b}$ & $i \mathrm{Bu}$ & 100 \\
$\mathbf{c}$ & $t \mathrm{Bu}$ & 92 \\
$\mathbf{d}$ & $\mathrm{Bn}$ & 100 \\
$\mathbf{e}$ & $\mathrm{H}$ & 0 \\
$\mathbf{f}$ & $\mathrm{Me}$ & 0
\end{tabular}

Scheme 2. Preparation of esters 2 and oxazolines $\mathbf{1}$ via $N$-Boc-amino alcohols.

hydrolysis. ${ }^{[6]}$ In our cases the proximity of the sulfonic acid moiety leads to the protonation of the amine functionality, making it a more suitable leaving group than the alcoholderived substituent. The macroscopic reverse of this chemistry accounts for the formation of $\mathbf{1}$ from 2 .<smiles>NCCOC(=O)c1ccccc1[S+](=O)[O-]</smiles><smiles>O=C(OCC/N=C/c1ccccc1O)c1ccccc1S(=O)(=O)[O-]</smiles>

\section{Crystallographic Studies}

Because of the unusual features observed in the preparation and hydrolysis of the oxazolines 1 we were anxious to confirm our structural assignments crystallographically. Fortunately, both the ester $\mathbf{2 a}$ and its derived oxazoline 1a could be obtained as single crystals from $\mathrm{MeCN}$ and $\mathrm{EtOH}$ respectively. Views of $\mathbf{1}$ and $\mathbf{2 a}$ are presented in Figure 1 and 2, respectively, which also give selected bond angles and distances, including hydrogen bonded $\mathrm{H}$-atoms. The structure of 1a confirms that the sulfonic acid oxazoline has been isolated in its zwitterionic form consistent with the observed reactivity for its formation and subsequent hydrolysis, although the bond lengths around C(7) suggest some residual double bond character to both the $\mathrm{O}$ and $\mathrm{N}$ atoms. The acidic $\mathrm{H}$ was located from difference Fourier synthesis and then refined as a part of a riding model $0.88 \AA$ from the oxazoline nitrogen, $1.83 \AA$ from the nearest $\mathrm{SO}_{3}$ oxygen. Relatively few chelating amino sulfonic acids have been subjected to crystallographic studies but these indicate similar zwitterionic structures with $\mathrm{N}-\mathrm{H}$ distances in the range 
$0.785-1.015 \AA .{ }^{[7]}$ The $\mathrm{O} \cdots \mathrm{H}$ distances $(2.05-2.511 \AA)$ in these structures are longer than those in 1a, while the $\mathrm{O} \cdots \mathrm{H}-\mathrm{N}$ angles $\left(100.3-139.4^{\circ}\right)$ are smaller than that of $\mathbf{1 a}$ $\left(153.7^{\circ}\right)$. Overall the placement of the NH proton in 1a is still, however, consistent with normal hydrogen bonding. In addition to confirming the atom connectivity of ester $\mathbf{2 a}$ is correctly assigned, Figure 2 shows (in contrast to purely intramolecular H-bonding in 1a), an extensive highly ordered hydrogen bonding network is present in $\mathbf{2}$.

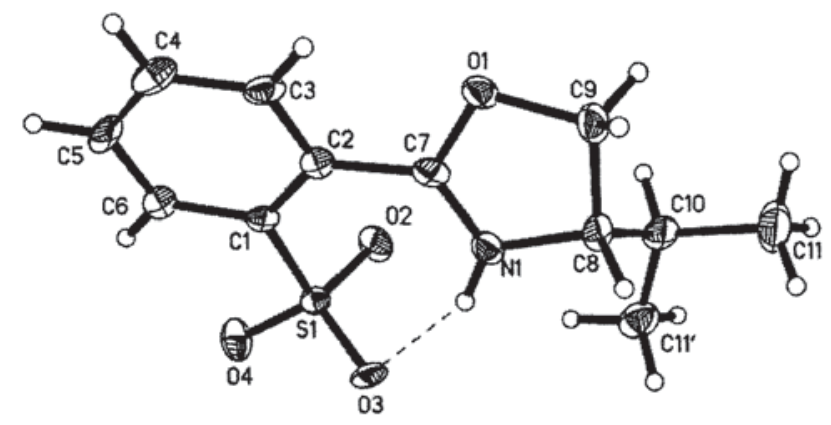

Figure 1. Molecular structure of 1a. Selected interatomic distances (A) and angles $\left(^{\circ}\right): \mathrm{S}(1)-\mathrm{O}(1) \quad 1.446(3), \quad \mathrm{S}(1)-\mathrm{O}(2) \quad 1.449(3)$, $\mathrm{S}(1)-\mathrm{O}(3)$ 1.471(3), $\mathrm{O}(1)-\mathrm{C}(7)$ 1.312(4), $\mathrm{O}(1)-\mathrm{C}(9)$ 1.478(4), $\mathrm{N}(1)-\mathrm{C}(7) 1.270(5), \mathrm{N}(1)-\mathrm{C}(8) 1.471(4), \mathrm{N}(1)-\mathrm{H}(1 \mathrm{~A}) \cdots \mathrm{O}(3) 1.83$, $\mathrm{N}(1) \cdots \mathrm{O}(3) 2.649(4), \mathrm{N}(1)-\mathrm{H}(1 \mathrm{~A})-\mathrm{O}(3) 153.7$

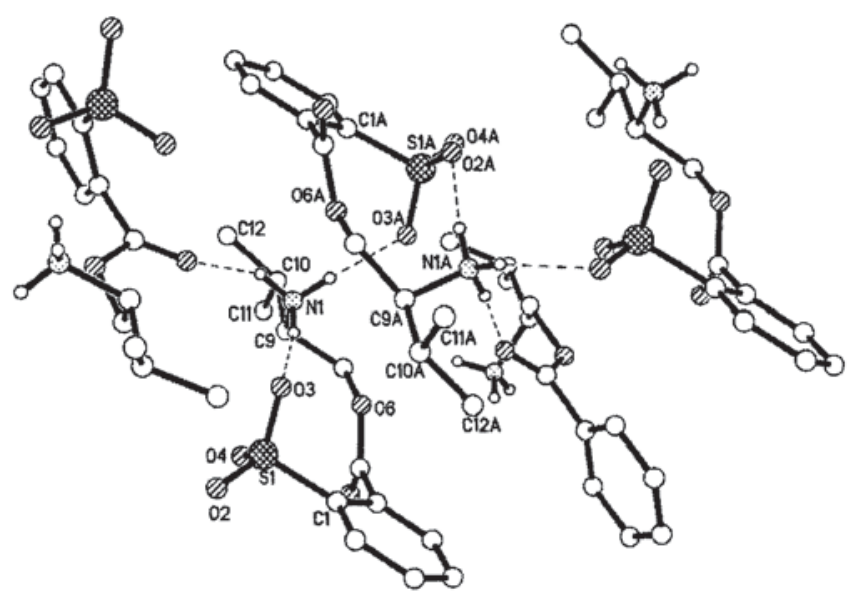

Figure 2. Packing diagram showing five molecules of 2a showing hydrogen bonding network, the two independent molecules in the structure are labelled. Selected H-bonding distances $(\AA)$ : $\mathrm{N}(1)-\mathrm{H}(1 \mathrm{~A}) \cdots \mathrm{O}(3) 1.93, \mathrm{~N}(1) \cdots \mathrm{O}(3) 2.824(3) ; \mathrm{N}(1)-\mathrm{H}(1 \mathrm{C}) \cdots \mathrm{O}(3 \mathrm{~A})$

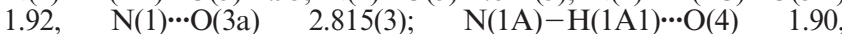

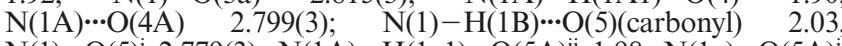
$\mathrm{N}(1) \cdots \mathrm{O}(5)^{\mathrm{i}} 2.779(3) ; \mathrm{N}(1 \mathrm{~A})-\mathrm{H}(1 \mathrm{a} 1) \cdots \mathrm{O}(5 \mathrm{~A})^{\mathrm{ii}} 1.98, \mathrm{~N}(1 \mathrm{a}) \cdots \mathrm{O}(5 \mathrm{~A})^{\mathrm{ii}}$ $2.887(3) ; \mathrm{i}=1-x, y-1 / 2,-z$; ii $=x-1, y, z$.

One potential use for the oxazoline ligands 1a is in asymmetric catalysis, therefore we were keen to confirm that this class of ligands can bind metal centres. Acetonitrile solutions of $1 \mathbf{a}$ were reacted with $\mathrm{M}(\mathrm{OAc})_{2}(\mathrm{M}=\mathrm{Pd}, \mathrm{Cu})$ at 90 ${ }^{\circ} \mathrm{C}$ and in the presence of 2,6-lutidine for copper(II) acetate. On cooling, yellow or dark blue crystals of 7 or 8 respectively formed. Mass spectrometry suggested that metathesis of the acetates had taken place to provide the bis-complex 7 in the case of palladium (Scheme 3). Elemental analysis results indicated identical behaviour for $\mathbf{8}$, but in this case no molecular ion could be observed.

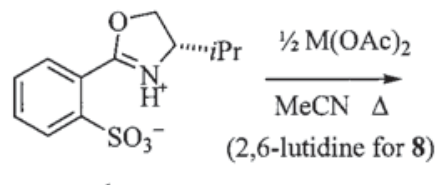

$1 \mathrm{a}$

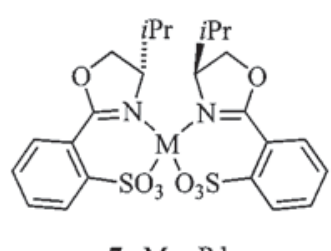

$7 \mathrm{M}=\mathrm{Pd}$

$8 \mathrm{M}=\mathrm{Cu}$
Scheme 3. Preparation of metal complexes of 1a.

X-ray crystallographic analysis confirmed the formation of $\mathrm{ML}_{2}$ complexes. The molecular structures of $\mathbf{7}$ and $\mathbf{8}$ are shown in Figure 3-4, respectively, together with selected bond lengths and angle data. The palladium complex 7 adopts an essencially perfect $\mathrm{d}^{8}$ square planar configuration and crystallised as an acetonitrile solvate. The $\mathrm{Pd}-\mathrm{O}$ distances and $\mathrm{Pd}-\mathrm{O}-\mathrm{S}$ are similar to those reported by Tulloch and co-workers for a Pd-OTs complex $2.123 \AA$ and $122.81^{\circ}$, respectively. ${ }^{[8]}$ The two ligands in 7 are inequivalent both in the solid state and in solution. For example, four methyl doublets at $\delta_{\mathrm{H}}=1.06,1.38,1.39$ and 1.62 are observed for the diastereotopic $i \operatorname{Pr}$ groups. The copper(II) complex adopts a distorted tetrahedral geometry. Again, the coordination of the sulfonic acids mimics those of known copper(II) tosylate species. ${ }^{[9]}$

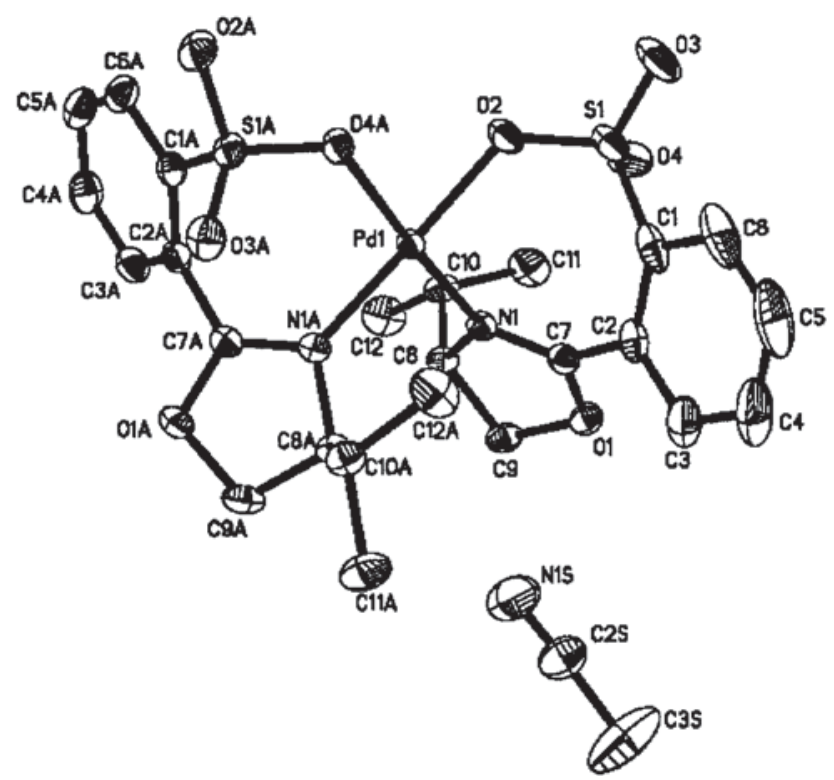

Figure 3. Molecular structure of 7. Selected interatomic distances (A) and angles ( $\left.{ }^{\circ}\right)$ : $\mathrm{Pd}(1)-\mathrm{O}(2) 2.016(13), \mathrm{Pd}(1)-\mathrm{O}(4 \mathrm{a}) 2.0334(11)$, $\operatorname{Pd}(1)-\mathrm{N}(1) \quad 1.9952(14), \quad \operatorname{Pd}(1)-\mathrm{N}(1 \mathrm{~A}) \quad 1.9908(15), \quad \mathrm{O}(2)-$ $\mathrm{Pd}-\mathrm{O}(4 \mathrm{~A})$ 83.22(6), O(2)-Pd-N(1) 93.25(6), O(4A)-Pd-N(1A) 94.52(6), $\quad \mathrm{N}(1)-\mathrm{Pd}-\mathrm{N}(1 \mathrm{~A}) \quad 89.03(6), \quad \mathrm{O}(2)-\mathrm{Pd}-\mathrm{N}(1)$ vs. $\mathrm{O}(4 \mathrm{~A})-\mathrm{Pd}-\mathrm{N}(1 \mathrm{~A})$ interplanar twist angle $1.1^{\circ}$. An acetonitrile of crystallisation is also shown in the plot.

\section{Reaction of Oxazolines 1 with Primary Amines}

Because of the versatility of sulfonamide units we wished to establish a simple derivatisation of the sulfonic acids $\mathbf{1}$ 


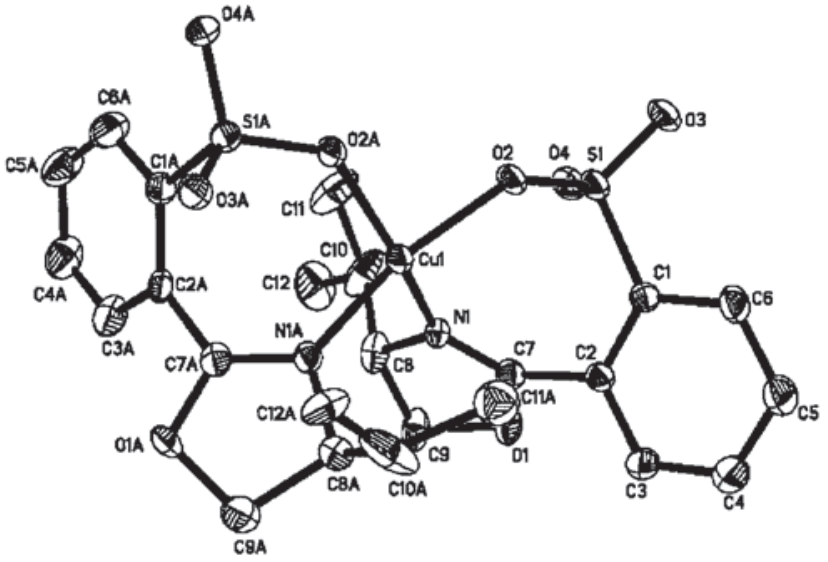

Figure 4. Molecular structure of $\mathbf{8}$. Selected interatomic distances (A) and angles( $\left(^{\circ}\right) \mathrm{Cu}(1)-\mathrm{O}(2)$ 1.940(3), $\mathrm{Cu}(1)-\mathrm{O}(2 \mathrm{a})$ 1.946(3), $\mathrm{Cu}(1)-\mathrm{N}(1) 1.947(4), \mathrm{Cu}(1)-\mathrm{N}(1 \mathrm{~A}) 1.942(3), \mathrm{O}(2)-\mathrm{Cu}-\mathrm{O}(2 \mathrm{~A})$ 86.80(12), $\mathrm{O}(2)-\mathrm{Cu}-\mathrm{N}(1) \quad 96.03(13), \quad \mathrm{O}(2 \mathrm{~A})-\mathrm{Cu}-\mathrm{N}(1 \mathrm{~A})$ 94.18(13), $\mathrm{N}(1)-\mathrm{Cu}-\mathrm{N}(1 \mathrm{~A}) \quad 92.13(14), \quad \mathrm{O}(2)-\mathrm{Cu}-\mathrm{N}(1)$ vs. $\mathrm{O}(2 \mathrm{~A})-\mathrm{Cu}-\mathrm{N}(1 \mathrm{~A})$ interplanar twist angle $31.4^{\circ}$.

to increase the diversity of our library. Perhaps the most simple method for sulfonamide formation is direct reaction with amines under thermal conditions leading to elimination of water. ${ }^{[10]}$ Reaction of $\mathbf{1 a}$ with $\mathrm{PhCH}_{2} \mathrm{NH}_{2}$ under Dean-Stark conditions $\left(\mathrm{PhCl}, 130{ }^{\circ} \mathrm{C}, 16 \mathrm{~h}\right)$, however, did not fashion the expected product 9 (Scheme 4). It was clear that a single new species is formed containing the reaction components and rearrangement chemistry seemed to have occurred.<smiles></smiles><smiles>[R16]N</smiles>

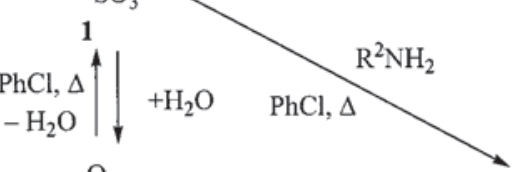<smiles>[R]C(C)COC(=O)c1ccccc1[S+]([O-])([O-])[O-]</smiles>
2<smiles>CC(C)C(CO)NC(=O)c1ccccc1S(=O)(=O)NCc1ccccc1</smiles>

11 (not formed)<smiles>CCCCC</smiles>

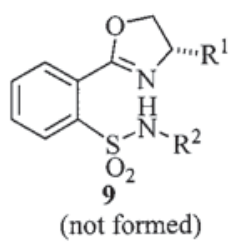<smiles>[R]NCC([R])NC(=O)c1ccccc1S(=O)(=O)O</smiles>

10

\begin{tabular}{c|c|l|l}
$\mathbf{1 0}$ & $\mathrm{R}^{1}$ & \multicolumn{1}{|c|}{$\mathrm{R}^{2}$} & Yield $/ \%$ \\
\hline $\mathbf{a}$ & $i \mathrm{Pr}$ & $\mathrm{Bn}$ & 65 \\
$\mathbf{b}$ & $i \mathrm{Bu}$ & $\mathrm{Bn}$ & 78 \\
$\mathbf{c}$ & $i \mathrm{Pr}$ & $\mathrm{CH}_{2}\left(1-\mathrm{C}_{10} \mathrm{H}_{7}\right)$ & 82 \\
$\mathbf{d}$ & $\mathrm{Bn}$ & $\mathrm{Bn}$ & 74 \\
$\mathbf{e}$ & $i \mathrm{Pr}$ & $R-\mathrm{CH}(\mathrm{Me}) \mathrm{Ph}$ & 73 \\
$\mathbf{f}$ & $i \mathrm{Bu}$ & $R-\mathrm{CH}(\mathrm{Me}) \mathrm{Ph}$ & 75
\end{tabular}

Scheme 4. Attempted preparation of sulfonamides via $\mathbf{1}$ or $\mathbf{2}$.

Both combustion analyses and mass spectra of the products indicated empirical formulas with no loss of $\mathrm{H}_{2} \mathrm{O}$. Confusingly, despite their proposed polar constitution these compounds could be isolated by chromatography on silica<smiles>[R]NCC1NC(=O)C([R])N1</smiles><smiles>[Te]=[Te]</smiles><smiles>[R]NCC([R])NC(=O)c1ccccc1S(=O)(=O)O</smiles>

10

Scheme 5. Formation of compounds $\mathbf{1 0}$

gel. Infra-red spectroscopic studies of $\mathbf{1 0}$ suggested the presence of an amide group $\left(1650-1620 \mathrm{~cm}^{-1}\right)$ and this is supported by the observation of an amido-like ${ }^{13} \mathrm{C}$ NMR resonance $\left(\delta_{C}=169.3-172.2\right)$ in each case. Proton NMR spectra of $\mathbf{1 0}$ clearly indicated the presence of three exchangable $X$-H protons (where $X=\mathrm{N}$ or $\mathrm{O}$ ) in each case. Reaction of the esters 2, under identical conditions also produced 10 (albeit in slightly lower yields of 50-60\%). Initial attempts at recrystallisation of 10a produced only fine needles unsuitable for X-ray analysis. Therefore compound 10a was subjected to extensive NMR studies in an attempt to define its structure. Exposure of a $\mathrm{CDCl}_{3}$ solution of 10a to $\mathrm{D}_{2} \mathrm{O}$ led to immediate replacement of two broad $1 \mathrm{H}$ signals at $\delta_{\mathrm{H}}=8.92$ and 8.90 (subsequently assigned to the $\mathrm{SO}_{3} \mathrm{H}$ and $\mathrm{NHR}^{2}$ resonances) and commensurate formation of an HOD signal at ca. $4.8 \mathrm{ppm}$. Further shaking with $\mathrm{D}_{2} \mathrm{O}$ led to replacement of a $1 \mathrm{H}$ doublet $(J=9.5 \mathrm{~Hz})$ at $\delta_{\mathrm{H}}=7.75$ (assigned to $\mathrm{CONH}$ ) over a 10 minute period. The ${ }^{1} \mathrm{H}:{ }^{1} \mathrm{H}$ COSY spectrum links the latter signal with a broad $\mathrm{CH}$, revealed through the COSY to be itself part of a $\mathrm{NHCH}(i \mathrm{Pr}) \mathrm{CH}_{2} \mathrm{OH}$ derived side chain. This led us to speculate initially that 10a was the hydrolysis product of the desired 9a (i.e. 11). The ${ }^{1} \mathrm{H}:{ }^{13} \mathrm{C}$ COSY spectrum of 10a showed a cross peak from the amido NH to the carbonyl signal at $\delta_{C}=172.2$ strongly suggesting that a carbonyl bound amide is formed. However, the proposed structure (11) could be discounted as it had already been prepared in complimentary studies by an independent route. ${ }^{[11]}$ Despite our best efforts we could not initially pin down the point of attachment of the $\mathrm{R}^{2} \mathrm{NH}_{2}$ derived amine unit. ${ }^{[12]}$ Fortunately, we were finally able to crystallise compound $\mathbf{1 0 c}$ from methanol and confirm the connectivity by an X-ray study (Figure 5).

The slow exchange of the sulfonamide $\mathrm{NHSO}_{2}$ with $\mathrm{D}_{2} \mathrm{O}$ and the fact that all examples of $\mathbf{1 0}$ may be isolated by chromatography on silica gel suggests strong hydrogen bonding in this class of compound. This is confirmed in the structure of 10c were the presence of intramolecular $\mathrm{H}$ bonding contacts between the amido $\mathrm{NH}$ and the sulfato group and also between the distal amine and the amide car- 


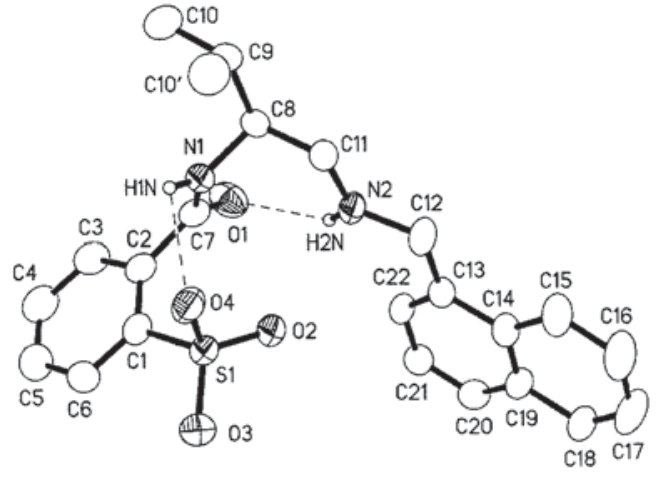

Figure 5. Molecular structure of 10c showing intramolecular hydrogen bonding. Selected $\mathrm{H}$-bond lengths $(\mathrm{A})$ and angles $\left({ }^{\circ}\right)$ : $\mathrm{N}(1)-\mathrm{H}(1) \mathrm{N} \cdots \mathrm{O}(4), \quad \mathrm{H}(1) \mathrm{N} \cdots \mathrm{O}(4) \quad 2.45, \quad \mathrm{~N}(1) \cdots \mathrm{O}(4) \quad 2.965(4)$, $\mathrm{N}(2)-\mathrm{H}(2) \mathrm{N} \cdots \mathrm{O}(1), \quad \mathrm{H}(2) \mathrm{N} \cdots \mathrm{O} 1 \quad 2.34, \quad \mathrm{~N}(2) \cdots \mathrm{O}(1) \quad 3.077(4)$, $\mathrm{N}(1)-\mathrm{H}(1) \mathrm{N} \cdots \mathrm{O}(4) 118, \mathrm{~N}(2)-\mathrm{H}(2) \mathrm{N} \cdots \mathrm{O}(1) 141$.

bonyl. Additionally, the unit cell of $\mathbf{1 0 c}$ contains methanol crystallisation solvent but this could not be modelled in terms of atomic sites. In $\mathrm{CD}_{3} \mathrm{OD}$ exchange of all the $\mathrm{OH}$ and $\mathrm{NH}$ protons in $\mathbf{1 0}$ is attained within minutes and spectra run in this solvent are devoid of signals due to these hydrogens. Hydrogen bonding effects also engenders compounds $\mathbf{1 0}$ to occlude other polar solvents unless strict anhydrous conditions are attained. For example, 10a, $\mathbf{d}$ and $\mathbf{f}$ readily crystallise as mono or hemi hydrates; a reproducible $\mathrm{H}_{2} \mathrm{O}$ signal at 1.9-2.0 ppm integrating to 1 or $2 \mathrm{H}$ can be detected in the ${ }^{1} \mathrm{H}$ (dried $\mathrm{CDCl}_{3}$ ) NMR spectra of these compounds in support of the analytical data. The formation of $\mathbf{1 0}$ can be most simply rationalised as an $\mathrm{S}_{\mathrm{N}} 2$ attack of the amine $\mathrm{R}^{2} \mathrm{NH}_{2}$ on the methylene of the oxazoline ring; the resulting fragmentation leading directly to the observed products (Scheme 5). Attempted sulfonamide formation from $\mathbf{1 0}$ by its exposure to further equivalents of $\mathrm{R}^{2} \mathrm{NH}_{2}\left(\mathrm{PhCl}, 130{ }^{\circ} \mathrm{C}, 16 \mathrm{~h}\right)$ led only to the recovery of $\mathbf{1 0}$.

\section{Conclusion}

The rapid synthesis of oxazolinesulfonic acids 1 has been demonstrated either through esters $\mathbf{2}$ or directly from 2-sulfobenzoic acid anhydride and $N$-Boc-protected amino alcohols. A useful displacement reaction of $\mathbf{1}$ or $\mathbf{2}$ has been discovered allowing simple access into diverse 2-amido-diamine benzenesulfonicacids 10. The method is eminently suitable for high throughput library synthesis, and investigations into the usefulness and application of such compounds in asymmetric catalysis is currently being undertaken both in our group and in collaboration with others.

\section{Experimental Section}

General: Procedures involving moisture sensitive reagents or intermediates were performed under atmospheres of argon or nitrogen using standard Schlenk techniques. Tetrahydrofuran and $\mathrm{Et}_{2} \mathrm{O}$ were distilled from sodium benzophenone ketyl. Other solvents were dried appropriately and stored over $4-\AA$ molecular sieves. Light petroleum refers to the fraction boiling in the range $40-60{ }^{\circ} \mathrm{C}$. Infrared spectra were recorded using a Perkin-Elmer 983G $(\mathrm{KBr}$ disc), Perkin-Elmer 882 or a Nicolet Avatar 360 FT-IR infrared spectrophotometer. The term "solid state" refers to direct analysis of the oil/solid, using a Nicolet Avatar 360 FT-IR reflecting probe. Proton and ${ }^{13} \mathrm{C}$ NMR spectra were recorded on either JEOL (GX 270) or Bruker (AM400, AV400 and DRX500) spectrometers at ambient temperature unless otherwise noted. Tetramethylsilane was used as an internal standard and $J$ values are given in Hz. Mass spectra were obtained on a Micromass 70E (electron-impact ionisation, EI+), Micromass LCT (electrospray ionization, ES+) and VG AutoSpec (fast-atom bomdardment, FAB+) machines. Specific rotations were measured using a Jasco DIP370 Digital polarimeter at ambient conditions and are given in units of $10^{-1{ }^{\circ}} \mathrm{cm}^{2} \mathrm{~g}^{-1} ;(c$ in $\mathrm{g} / 100 \mathrm{~mL}$ ). Boc-amino alcohols were prepared by literature routes. ${ }^{[13]}$ Commercially supplied 2-sulfobenzoic acid anhydride proved unsatisfactory; it was sublimed $\left(170^{\circ} \mathrm{C}, 0.1\right.$ Torr $)$ before use.

Representative Procedure. 2-[(S)-4-Isopropyl-4,5-dihydrooxazol-2yl]benzenesulfonic Acid (1a): Chlorobenzene $(15 \mathrm{~mL})$ was added to 2a (180 mg, $0.63 \mathrm{mmol})$ and the reaction mixture refluxed with stirring using a Dean-Stark trap ( $5 \mathrm{~mL}$ capacity) for $16 \mathrm{~h}$. Upon completion the reaction was concentrated under vacuum to give 1a $(168 \mathrm{mg} ; 100 \%)$ as a white solid, with no further purification required. ${ }^{[14]}$ M.p. $226-227{ }^{\circ} \mathrm{C}(\mathrm{EtOH}) .[\alpha]_{\mathrm{D}}=-44.2(c=3.89$, $\mathrm{MeOH}) .{ }^{1} \mathrm{H}$ NMR $\left(400 \mathrm{MHz}, \mathrm{CD}_{3} \mathrm{OD}\right): \delta=8.20(\mathrm{dd}, 1 \mathrm{H}, J=$ 8.0, 1.0; Ar), 7.98 (dd, $1 \mathrm{H}, J=8.0,1.0 ;$ Ar), 7.91 (dt, $1 \mathrm{H}, J=$ 8.0, 1.0; Ar), 7.76 (dt, $1 \mathrm{H}, J=8.0,1.0 ; \mathrm{Ar}), 5.26$ (apparent $\mathrm{t}, 1$ $\mathrm{H}, J=9.8), 5.04$ (dd, $1 \mathrm{H}, J=9.8,7.4), 4.54(\mathrm{~m}, 1 \mathrm{H}), 2.17(\mathrm{~m}, 1$ $\left.\mathrm{H}, \mathrm{C} H \mathrm{Me}_{2}\right), 1.17$ (d, $\left.3 \mathrm{H}, J=6.7 ; M e\right), 1.11(\mathrm{~d}, 3 \mathrm{H}, J=6.7 ; M e)$ ppm, H/D exchange in $\mathrm{SO}_{3} \mathrm{H}$ group with $\mathrm{D}$ from solvent. ${ }^{13} \mathrm{C}$ NMR $\left(67.8 \mathrm{MHz}, \mathrm{CD}_{3} \mathrm{OD}\right): \delta=174.8\left(\mathrm{C}_{o x}\right), 146.9,136.3,132.2$, 131.9, 129.7, 119.2, 77.8, 66.6, 32.9, $18.2(\mathrm{Me}), 18.0(\mathrm{Me}) \mathrm{ppm}$. IR (solid state): $\tilde{v}=1659 \mathrm{~s}\left(\mathrm{C}=\mathrm{N}_{\mathrm{ox}}\right), 1501 \mathrm{~m}, 1456 \mathrm{w}, 1340 \mathrm{w}, 1254$ vs, 1185 vs, $1145 \mathrm{~m}, 1128 \mathrm{~m}, 1078 \mathrm{~m}, 1044 \mathrm{w}, 1016 \mathrm{~s}, 935 \mathrm{~m}, 800$ m, $759 \mathrm{~m}, 736 \mathrm{~s}, 691 \mathrm{~m} \mathrm{~cm}^{-1}$. MS: $m / z(\mathrm{ES}+): 846\left(\mathrm{M}_{3} \mathrm{H}^{+}(\mathrm{MeCN})\right.$, $60 \%), 270\left(\mathrm{MH}^{+}, 100\right) . \mathrm{C}_{12} \mathrm{H}_{15} \mathrm{NO}_{4} \mathrm{~S}$ (269.32): calcd. C 53.5, H 5.6, $\mathrm{N}$ 5.2; found C 53.6, H 5.6, N 5.1.

Compound 1a could also be prepared by direct reaction of 2-sulfobenzoic acid anhydride $(1.50 \mathrm{~g}, 8.14 \mathrm{mmol})$ and L-valinol $(0.84 \mathrm{~g}$, $8.14 \mathrm{mmol})$ in $\mathrm{PhCl}(50 \mathrm{~mL})$ (Dean-Stark apparatus, reflux $17 \mathrm{~h})$. Evaporation of the $\mathrm{PhCl}$ under vacuum followed by recrystallisation of the residue from anhydrous ethanol afforded $1.25-1.32 \mathrm{~g}$ $(55-60 \%)$. This alternative procedure was, however, ineffective for compounds $\mathbf{1 b}-\mathbf{e}$.

2-((S)-4-Isobutyl-4,5-dihydrooxazol-2-yl)benzenesulfonic Acid (1b): Prepared from 2b $(238 \mathrm{mg}, 0.79 \mathrm{mmol})$, recrystallisation from EtOH gave $\mathbf{1 b}(220 \mathrm{mg}, 98 \%)$ as white crystals. M.p. $201-202{ }^{\circ} \mathrm{C}$ $(\mathrm{EtOH}) .[\alpha]_{\mathrm{D}}=-13.8(c=1.0, \mathrm{EtOH}) .{ }^{1} \mathrm{H}$ NMR $(400 \mathrm{MHz}$, $\left.\mathrm{CD}_{3} \mathrm{OD}\right): \delta=8.18(\mathrm{dd}, 1 \mathrm{H}, J=8.0,1.0 ; \mathrm{Ar}), 7.94(\mathrm{dd}, 1 \mathrm{H}, J=$ 8.0, 1.0; Ar), 7.88 (dt, $1 \mathrm{H}, J=8.0,1.0 ; \mathrm{Ar}), 7.76$ (dt, $1 \mathrm{H}, J=$ 8.0, 1.0; $\mathrm{Ar}), 5.33\left(\mathrm{dd}, 1 \mathrm{H}, J=8.8,7.8 ; \mathrm{OCH}_{2 \alpha}\right), 4.88(\mathrm{dd}, 1 \mathrm{H}$, $\left.J=8.8,7.0 ; \mathrm{OCH}_{2 \beta}\right), 4.86-4.79(\mathrm{~m}, 1 \mathrm{H}, \mathrm{CHN}), 1.99(\mathrm{ddd}, 1 \mathrm{H}$, $J=13.5,7.6,6.0), 1.88\left(\mathrm{~m}, 1 \mathrm{H}, \mathrm{C} H \mathrm{Me}_{2}\right), 1.75$ (ddd, $1 \mathrm{H}, J=$ $13.5,7.9,6.0), 1.07$ (d, $3 \mathrm{H}, J=6.5 ; M e), 1.06$ (d, $3 \mathrm{H}, J=6.5$; $\mathrm{Me}$ ) ppm, $\mathrm{H} / \mathrm{D}$ exchange in $\mathrm{SO}_{3} \mathrm{H}$ group with $\mathrm{D}$ from solvent. ${ }^{13} \mathrm{C}$ NMR (100 MHz, CD $3 \mathrm{OD}): \delta=174.8\left(\mathrm{C}_{o x}\right), 147.1(\mathrm{C}), 136.2$, 132.0, 131.8, 129.7, 119.4 (C), 80.0, 59.7, 43.6, 26.2, 23.0 (Me), 22.1 (Me) ppm. IR (solid state): $\tilde{v}=1651 \mathrm{~s}\left(\mathrm{C}=\mathrm{N}_{\mathrm{ox}}\right), 1497 \mathrm{w}, 1451 \mathrm{w}$, 1356 w, 1246 vs, 1190 vs, 1172 s, 1133 m, 1078 w, 1018 s, 939 m, $837 \mathrm{w}, 792 \mathrm{~m}, 751 \mathrm{~m}, 737 \mathrm{~s}, 697 \mathrm{~cm}^{-1}$. MS: $\mathrm{m} / \mathrm{z}(\mathrm{ES}+): 284\left(\mathrm{MH}^{+}\right.$, 
$100 \%$ ); found (HRMS): $\mathrm{MH}^{+}$284.0982, $\mathrm{C}_{13} \mathrm{H}_{18} \mathrm{NO}_{4} \mathrm{~S}$ requires 284.0957.

2-((S)-4-tert-Butyl-4,5-dihydrooxazol-2-yl)benzenesulfonic Acid (1c): Prepared from 2c $(301 \mathrm{mg}, 1.00 \mathrm{mmol})$, recrystallisation from EtOH gave 1c (260 mg, 92\%) as white crystals. M.p. $>240{ }^{\circ} \mathrm{C}(\mathrm{dec}$., EtOH $) .[\alpha]_{\mathrm{D}}=-23.1(c=1.0, \mathrm{EtOH}) .{ }^{1} \mathrm{H} \mathrm{NMR}(400 \mathrm{MHz}$, $\left.\mathrm{CD}_{3} \mathrm{OD}\right): \delta=8.24$ (dd, $\left.1 \mathrm{H}, J=7.7,1.0 ; \mathrm{Ar}\right), 8.07$ (dd, $1 \mathrm{H}, J=$ 7.7, 1.0; Ar), 7.93 (dt, $1 \mathrm{H}, J=7.7,1.0$; Ar), 7.78 (dt, $1 \mathrm{H}, J=$ 7.7, 1.0; Ar), 5.19 (dd, $1 \mathrm{H}, J=10.4,9.9 ; \mathrm{OCH}_{2 \alpha}$ ), overlapped by $5.15(\mathrm{dd}, 1 \mathrm{H}, J=9.9,7.5 ; \mathrm{C} H \mathrm{~N}), 4.60(\mathrm{dd}, 1 \mathrm{H}, J=10.4,7.5$; $\left.\mathrm{OCH}_{2 \beta}\right), 1.14(\mathrm{~s}, 9 \mathrm{H}, t \mathrm{Bu}) \mathrm{ppm}, \mathrm{H} / \mathrm{D}$ exchange in $\mathrm{SO}_{3} H$ group with D from solvent. ${ }^{13} \mathrm{C}$ NMR $\left(100 \mathrm{MHz}, \mathrm{CD}_{3} \mathrm{OD}\right): \delta=174.4$ $\left(\mathrm{C}_{o x}\right), 147.1(\mathrm{C}), 136.9,132.9,132.1,130.0,118.8$ (C), 76.0, 70.0, $35.1(\mathrm{Bu}), 25.2(\mathrm{Bu}) \mathrm{ppm}$. IR (solid state): $\tilde{v}=1655 \mathrm{~s}\left(\mathrm{C}=\mathrm{N}_{\mathrm{ox}}\right)$, $1497 \mathrm{~m}, 1452 \mathrm{w}, 1379 \mathrm{w}, 1255 \mathrm{vs}, 1180$ vs, $1131 \mathrm{~m}, 1082 \mathrm{w}, 1017 \mathrm{~s}$, 928 w, 901 w, 749 s, 736 s, $687 \mathrm{~cm}^{-1}$. MS: $m / z(\mathrm{ES}+): 284\left(\mathrm{MH}^{+}\right.$ $100 \%$ ); found (HRMS): $\mathrm{MH}^{+} 284.0967, \mathrm{C}_{13} \mathrm{H}_{18} \mathrm{NO}_{4} \mathrm{~S}$ requires 284.0957. $\mathrm{C}_{13} \mathrm{H}_{17} \mathrm{NO}_{4} \mathrm{~S}$ (283.34): calcd. C 55.1, H 6.05, N 4.9; found C 55.2, H 6.1, N 4.8 .

2-((S)-4-Benzyl-4,5-dihydrooxazol-2-yl)benzenesulfonic Acid (1d): Prepared from $2 \mathbf{d}(0.99 \mathrm{~g}, 2.96 \mathrm{mmol})$, recrystallisation from dichloromethane/light petroleum ether gave 1d $(807 \mathrm{mg}, 86 \%)$ as white crystals. M.p. $190-191{ }^{\circ} \mathrm{C}(\mathrm{EtOH}) .[\alpha]_{\mathrm{D}}=-53.2(c=1.0$, EtOH). ${ }^{1} \mathrm{H}$ NMR (400 MHz, $\left.\mathrm{CD}_{3} \mathrm{OD}\right): \delta=8.16(\mathrm{~d}, 1 \mathrm{H}, J=7.8$, 1.0; Ar), 7.88 (dt, $1 \mathrm{H}, J=7.8,1.2 ; \mathrm{Ar}), 7.79$ (dd, $1 \mathrm{H}, J=7.8$, 1.0; Ar), 7.71 (dt, $1 \mathrm{H}, J=7.8,1.2$; Ar), 7.42-7.34 (m, $5 \mathrm{H}, \mathrm{Ar}$ ), 5.27 (apparent t, $\left.1 \mathrm{H}, J=12.1 ; \mathrm{OCH}_{2 \alpha}\right), 5.08-5.02(\mathrm{~m}, 2 \mathrm{H}$, $\mathrm{OCH}_{2 \beta}$ and $\mathrm{CHN}$ ), 3.29 (dd, $\left.1 \mathrm{H}, J=13.9,5.3 ; \mathrm{CH}_{2 \alpha} \mathrm{Ph}\right), 3.22$ $\left(\mathrm{dd}, 1 \mathrm{H}, J=13.9,6.8 ; \mathrm{CH}_{2 \beta} \mathrm{Ph}\right) \mathrm{ppm}, \mathrm{H} / \mathrm{D}$ exchange in $\mathrm{SO}_{3} H$ group with D from solvent. ${ }^{13} \mathrm{C}$ NMR $\left(100 \mathrm{MHz}, \mathrm{CD}_{3} \mathrm{OD}\right): \delta=$ $175.4\left(\mathrm{C}_{o x}\right), 146.7(\mathrm{C}), 136.3,135.9,131.7,131.6,130.7,130.6$, 129.6, 128.6, $119.4(\mathrm{C}), 79.0,62.2,40.0 \mathrm{ppm}$. IR (solid state): $\tilde{v}=$ $1653 \mathrm{~s}\left(\mathrm{C}=\mathrm{N}_{\mathrm{ox}}\right), 1574 \mathrm{w}, 1499 \mathrm{~s}, 1454 \mathrm{~m}, 1345 \mathrm{w}, 1255 \mathrm{vs}, 1184 \mathrm{vs}$, $1146 \mathrm{~m}, 1130 \mathrm{~m}, 1020 \mathrm{~s}, 934 \mathrm{~m}, 786 \mathrm{~m}, 733 \mathrm{~s}, 699 \mathrm{~s} \mathrm{~cm}^{-1}$. MS $\mathrm{m} / \mathrm{z}(\mathrm{ES}+): 318\left(\mathrm{MH}^{+}, 100 \%\right)$; found (HRMS): $\mathrm{MH}^{+} 318.0746$, $\mathrm{C}_{16} \mathrm{H}_{15} \mathrm{NSO}_{4}$ requires 318.0742. $\mathrm{C}_{16} \mathrm{H}_{17} \mathrm{NO}_{5} \mathrm{~S}$ (317.36): calcd. C 60.55, H 4.8, N 4.4; found C 60.15, H 4.65, N 4.3.

Representative Procedure. (S)-2-Amino-3-methylbutyl 2-Sulfobenzoate (2a): To a stirred solution of 2-sulfobenzoic acid cyclic anhydride $4(3.73 \mathrm{~g}, 20.3 \mathrm{mmol})$ in dry $\mathrm{MeCN}(40 \mathrm{~mL})$ under inert atmosphere, was added $N$-Boc-L-valinol $(4.12 \mathrm{~g}, 20.3 \mathrm{mmol})$ as a solution in dry $\mathrm{MeCN}(10 \mathrm{~mL})$. The reaction mixture was refluxed for $8 \mathrm{~h}$, a white insoluble precipitate being formed during the course of the reaction. The reaction mixture was allowed to cool to room temperature and stirred overnight. The reaction was cooled to $0{ }^{\circ} \mathrm{C}$ in the freezer for $12 \mathrm{~h}$, the white insoluble product was filtered off, washed with light petroleum ether then dried under high vacuum to give 2a $(3.91 \mathrm{~g}, 67 \%)$ as a white solid. An additional experiment gave (1.68 g, 87\%). M.p. $208-209^{\circ} \mathrm{C}(\mathrm{EtOH})$. $[\alpha]_{\mathrm{D}}=+36.3(c=1.0, \mathrm{EtOH}) .{ }^{1} \mathrm{H}$ NMR $\left(400 \mathrm{MHz}, \mathrm{CD}_{3} \mathrm{OD}\right): \delta=$ $8.05(\mathrm{dt}, 1 \mathrm{H}, J=7.5,1.0), 7.67-7.57(\mathrm{~m}, 3 \mathrm{H}), 4.80(\mathrm{dd}, 1 \mathrm{H}, J=$ $12.1,3.0 ; \mathrm{OCH}_{2 \alpha}$ ), 4.28 (dd, $1 \mathrm{H}, J=12.1,9.5 ; \mathrm{OCH}_{2 \beta}$ ), 3.47 (ddd, $1 \mathrm{H}, J=9.5,7.1,3.0 ; \mathrm{C} H \mathrm{~N}), 2.08(\mathrm{~d}$ sept, $1 \mathrm{H}, J=7.1,6.9$, $\left.\mathrm{C} H \mathrm{Me}_{2}\right), 1.33(\mathrm{~d}, 3 \mathrm{H}, J=6.9 ; M e), 1.14(\mathrm{~d}, 3 \mathrm{H}, J=6.9 ; M e)$ ppm, $\mathrm{H} / \mathrm{D}$ exchange in $\mathrm{OH}$ and $\mathrm{NH}_{2}$ groups with $\mathrm{D}$ from solvent. ${ }^{13} \mathrm{C}$ NMR (100 MHz, $\left.\mathrm{CD}_{3} \mathrm{OD}\right): \delta=169.8(\mathrm{CO}), 144.0,132.1$, 132.0, 131.6, 130.0, 129.0, $64.7\left(\mathrm{CH}_{2}\right), 57.1(\mathrm{CH}), 29.6(\mathrm{CH}), 19.1$ $\left(\mathrm{CH}_{3}\right), 18.8\left(\mathrm{CH}_{3}\right)$ ppm. IR ( $\mathrm{KBr}$ disc): $\tilde{v}=3449 \mathrm{~m}, 3157 \mathrm{~m}, 2966$ $\mathrm{m}, 1736$ vs $\left(\mathrm{C}=\mathrm{O}_{\text {ester }}\right), 1618 \mathrm{~m}, 1519 \mathrm{~s}, 1300 \mathrm{~s}, 1243 \mathrm{~s}, 1226 \mathrm{~s}, 1198$ m, 1179 s, $1122 \mathrm{~m}, 1082 \mathrm{~s}, 1017 \mathrm{~s}, 782 \mathrm{~m}, 762 \mathrm{~m}, 740 \mathrm{~m}, 662 \mathrm{~m}$, $617 \mathrm{~s} \mathrm{~cm}^{-1}$. MS: $\mathrm{m} / \mathrm{z}(\mathrm{ES}+)$ : $862\left(\mathrm{M}_{3} \mathrm{H}^{+}, 100 \%\right), 575\left(\mathrm{M}_{2} \mathrm{H}^{+}, 50\right)$, $288\left(\mathrm{MH}^{+}, 10\right)$; found (HRMS): $\mathrm{MH}^{+} 288.0923, \mathrm{C}_{12} \mathrm{H}_{18} \mathrm{NO}_{5} \mathrm{~S}$ re- quires 288.0906. Other compounds were formed in an analogous manner. $\mathrm{C}_{12} \mathrm{H}_{17} \mathrm{NO}_{5} \mathrm{~S}$ (287.33): calcd. C 50.2, H 6.0, N 4.9; found C 50.3, H 6.0, N 4.8 .

(S)-2-Amino-4-methylpentyl 2-Sulfobenzoate (2b): Prepared from $\mathrm{N}$ Boc-L-leucinol (500 mg, $2.30 \mathrm{mmol}$ ), and 4 (422 mg, $2.30 \mathrm{mmol}$ ). Purification by recrystallisation from $\mathrm{MeCN}$ gave $\mathbf{2 b}(470 \mathrm{mg}, 68 \%)$ as a white solid. M.p. $231-232{ }^{\circ} \mathrm{C}(\mathrm{MeCN}) \cdot[\alpha]_{\mathrm{D}}=+22.2(\mathrm{c}=$ 1.0, EtOH). ${ }^{1} \mathrm{H}$ NMR (400 MHz, $\left.\mathrm{CD}_{3} \mathrm{OD}\right): \delta=8.05$ (dd, $1 \mathrm{H}, J=$ 7.0, 1.0; Ar), 7.67-7.57 (m, $3 \mathrm{H}, \mathrm{Ar}), 4.74$ (dd, $1 \mathrm{H}, J=12.2,3.0$; $\mathrm{OCH}_{2 \alpha}$ ), 4.19 (dd, $\left.1 \mathrm{H}, J=12.2,9.0 ; \mathrm{OCH}_{2 \beta}\right), 3.75$ (ddd, $1 \mathrm{H}, J=$ 9.0, 7.0, 3.0; $\mathrm{CHN}$ ), 1.81 (sept, $\left.1 \mathrm{H}, J=7.0 ; \mathrm{CHMe}_{2}\right), 1.67-1.52$ $\left(\mathrm{m}, 2 \mathrm{H}, \mathrm{CHCH}_{2} i \mathrm{Pr}\right.$ ), 1.04 (d, $\left.3 \mathrm{H}, J=7.0 ; M e\right), 1.03$ (d, $3 \mathrm{H}, J=$ $7.0 ; \mathrm{Me}) \mathrm{ppm}, \mathrm{H} / \mathrm{D}$ exchange in $\mathrm{OH}$ and $\mathrm{NH}_{2}$ groups with $\mathrm{D}$ from solvent. ${ }^{13} \mathrm{C}$ NMR $\left(100 \mathrm{MHz}, \mathrm{CD}_{3} \mathrm{OD}\right): \delta=169.8$ (CO), 143.9, 132.2, 132.1, 131.7, 130.0, 129.0, $65.9\left(\mathrm{CH}_{2}\right), 50.1(\mathrm{CH}), 39.2$ $\left(\mathrm{CH}_{2}\right), 25.6(\mathrm{CH}), 22.9\left(\mathrm{CH}_{3}\right), 22.5\left(\mathrm{CH}_{3}\right) \mathrm{ppm}$. IR (solid state): $\tilde{v}=1736 \mathrm{~m}\left(\mathrm{C}=\mathrm{O}_{\text {ester }}\right), 1693 \mathrm{~m}, 1523 \mathrm{~m}, 1327 \mathrm{~m}, 1216 \mathrm{~m}, 1293 \mathrm{~m}$, 1242 vs, 1228 s, 1188 s, 1175 vs, 1142 s, 1118 w, 1081 s, 1017 vs, 754 vs $\mathrm{cm}^{-1}$. MS: $\mathrm{m} / \mathrm{z}(\mathrm{ES}+)$ : $904\left(\mathrm{M}_{3} \mathrm{H}^{+}, 100 \%\right), 603\left(\mathrm{M}_{2} \mathrm{H}^{+}, 30\right), 302$ $\left(\mathrm{MH}^{+}, 30\right.$ ); found (HRMS): $\mathrm{MH}^{+} 302.0994, \mathrm{C}_{13} \mathrm{H}_{20} \mathrm{NO}_{5} \mathrm{~S}$ requires 302.0984. $\mathrm{C}_{13} \mathrm{H}_{19} \mathrm{NO}_{5} \mathrm{~S}$ (301.36): calcd. C 51.8, H 6.4, N 4.65; found $\mathrm{C} 51.5, \mathrm{H} 6.4, \mathrm{~N} 4.3$.

(S)-2-Amino-3,3-dimethylbutyl 2-Sulfobenzoate (2c): Prepared from $N$-Boc-L-tert-leucinol $(588 \mathrm{mg}, \quad 2.70 \mathrm{mmol})$, and $4(496 \mathrm{mg}$, $2.70 \mathrm{mmol}$ ). Purification by recrystallisation from cold $\mathrm{MeCN}$ gave 2c $(700 \mathrm{mg}, 87 \%)$ as a white crystalline solid. M.p. $225-226^{\circ} \mathrm{C}$ $(\mathrm{MeCN}) \cdot[\alpha]_{\mathrm{D}}=-25.6\left(c=1.0, \mathrm{CHCl}_{3}\right) .{ }^{1} \mathrm{H} \mathrm{NMR}(400 \mathrm{MHz}$, $\left.\mathrm{CD}_{3} \mathrm{OD}\right): \delta=8.05(\mathrm{dd}, 1 \mathrm{H}, J=7.0,1.0 ; \mathrm{Ar}), 7.65-7.59(\mathrm{~m}, 3 \mathrm{H}$, Ar), 4.89 (dd, $\left.1 \mathrm{H}, J=12.0,3.0 ; \mathrm{OCH}_{2 \alpha}\right), 4.26$ (dd, $1 \mathrm{H}, J=12.0$, 10.6; $\mathrm{OCH}_{2 \beta}$ ), 3.49 (dd, $1 \mathrm{H}, J=10.6,3.0 ; \mathrm{C} H \mathrm{~N}$ ), 1.14 (s, $9 \mathrm{H}$, $t \mathrm{Bu}) \mathrm{ppm}, \mathrm{H} / \mathrm{D}$ exchange in $\mathrm{OH}$ and $\mathrm{NH}_{2}$ groups with $\mathrm{D}$ from solvent. ${ }^{13} \mathrm{C}$ NMR $\left(100 \mathrm{MHz}, \mathrm{CD}_{3} \mathrm{OD}\right): \delta=169.8(\mathrm{CO}), 144.0$, 132.1, 132.0, 131.6, 130.0, 129.0, $64.1\left(\mathrm{CH}_{2}\right), 60.2(\mathrm{CH}), 33.1(\mathrm{C})$, $26.6\left(\mathrm{CH}_{3}\right) \mathrm{ppm}$. IR (solid state): $\tilde{v}=2970 \mathrm{w}, 1728 \mathrm{~s}\left(\mathrm{C}=\mathrm{O}_{\text {ester }}\right)$, $1633 \mathrm{w}, 1528 \mathrm{~m}, 1303 \mathrm{w}, 1280 \mathrm{~s}, 1245$ vs, 1229 s, 1180 vs, 1171 vs, 1147 s, 1118 w, 1109 s, 1082 vs, 1020 vs, $780 \mathrm{~m}, 758 \mathrm{~s}, 739 \mathrm{~m} \mathrm{~cm}^{-1}$. MS: $m / z(\mathrm{ES}+)$ : $904\left(\mathrm{M}_{3} \mathrm{H}^{+}, 100 \%\right), 603\left(\mathrm{M}_{2} \mathrm{H}^{+}, 30\right), 302\left(\mathrm{MH}^{+}\right.$, 30); found (FAB + , HRMS): $\mathrm{MH}^{+} 302.1081, \mathrm{C}_{13} \mathrm{H}_{20} \mathrm{NO}_{5} \mathrm{~S}$ requires 302.1062. $\mathrm{C}_{13} \mathrm{H}_{19} \mathrm{NO}_{5} \mathrm{~S}$ (301.36): calcd. C 51.8, H 6.4, N 4.7; found C 51.6, H 6.4, N 4.7.

(S)-2-Amino-3-phenylpropyl 2-Sulfobenzoate (2d): Prepared from $\mathrm{N}$ Boc-L-phenylalaninol (2.01 g, $8.0 \mathrm{mmol})$, and 4 (1.47 g, $8.0 \mathrm{mmol})$. Purification by recrystallisation from cold $\mathrm{MeCN}$ gave $2 \mathrm{~d}(2.57 \mathrm{~g}$, $96 \%)$ as a white powder. Subsequent recrystallisation from EtOH/ $\mathrm{H}_{2} \mathrm{O}$ gave $2 \mathrm{~d}(1.69 \mathrm{~g}, 63 \%)$ as off-white crystals. M.p. $174-175{ }^{\circ} \mathrm{C}$ $(\mathrm{EtOH}) .[\alpha]_{\mathrm{D}}=+40.0(c=1.0, \mathrm{EtOH}) .{ }^{1} \mathrm{H}$ NMR $(400 \mathrm{MHz}$, $\mathrm{CD}_{3} \mathrm{OD}$ ): $\delta=8.06$ (d, $1 \mathrm{H}, J=7.5$; Ar), 7.66-7.56 (m, $3 \mathrm{H}, \mathrm{Ar}$ ), 7.40-7.30 (m, $5 \mathrm{H}, \mathrm{Ar}), 4.63$ (dd, $1 \mathrm{H}, J=12.1,2.9$; $\mathrm{OCH}_{2 \alpha}$ ), 4.22 $\left(\mathrm{dd}, 1 \mathrm{H}, J=12.1,7.8 ; \mathrm{OCH}_{2 \beta}\right), 3.94-3.88(\mathrm{~m}, 1 \mathrm{H}, \mathrm{CHN}), 3.08$ (apparent dd, $2 \mathrm{H}, J=8.5,4.5 ; \mathrm{CH}_{2} \mathrm{Ph}$ ) ppm, H/D exchange in $\mathrm{OH}$ and $\mathrm{NH}_{2}$ groups with $\mathrm{D}$ from solvent. ${ }^{13} \mathrm{C} \mathrm{NMR}(67.8 \mathrm{MHz}$, $\left.\mathrm{CD}_{3} \mathrm{OD}\right): \delta=169.6(\mathrm{CO}), 143.9,136.4,132.0,131.9,131.6,130.3$, 130.1, 130.0, 129.0, 128.6, $65.0\left(\mathrm{CH}_{2}\right), 53.0(\mathrm{CH}), 36.3\left(\mathrm{CH}_{2}\right) \mathrm{ppm}$. IR (solid state): $\tilde{v}=3489 \mathrm{w}, 3288 \mathrm{w}, 1751 \mathrm{~s}\left(\mathrm{C}=\mathrm{O}_{\text {ester }}\right), 1618 \mathrm{w}$, $1524 \mathrm{~m}, 1291 \mathrm{~s}, 1255 \mathrm{~s}, 1239 \mathrm{~m}, 1217 \mathrm{~s}, 1190 \mathrm{vs}, 1145 \mathrm{~m}, 1124 \mathrm{~s}$, $1081 \mathrm{~s}, 1018 \mathrm{~s}, 981 \mathrm{~m}, 788 \mathrm{~m}, 759 \mathrm{~s}, 788 \mathrm{~m}, 740 \mathrm{~m}, 720 \mathrm{w}, 706 \mathrm{~s}$ $\mathrm{cm}^{-1}$. MS: $m / z(\mathrm{ES}+)$ : $671\left(\mathrm{M}_{2} \mathrm{H}^{+}, 40 \%\right), 336\left(\mathrm{MH}^{+}, 100\right)$; found (ES+, HRMS): $\mathrm{MH}^{+}$336.0881, $\mathrm{C}_{16} \mathrm{H}_{18} \mathrm{NO}_{5} \mathrm{~S}$ requires 336.0906]. $\mathrm{C}_{16} \mathrm{H}_{17} \mathrm{NO}_{5} \mathrm{~S} \cdot \mathrm{H}_{2} \mathrm{O}$ (335.38): calcd. C 54.4, $\mathrm{H}$ 5.4, N 4.0; found $\mathrm{C}$ 54.0, H 5.3, N 3.8 .

2-Aminoethyl 2-Sulfobenzoate (2e): Prepared from $N$-Boc-ethanolamine $(4.61 \mathrm{~g}, 28.6 \mathrm{mmol})$, and 4 (5.26 g, $28.6 \mathrm{mmol})$. Precipitated 
directly from $\mathrm{MeCN}$ to give $2 \mathrm{e}(6.60 \mathrm{~g}, 94 \%)$ as a white solid. M.p. $>250{ }^{\circ} \mathrm{C}$ (dec., $\left.\mathrm{MeCN}\right)$. IR (solid state): $\tilde{\mathrm{v}}=3179 \mathrm{w}, 1728 \mathrm{~s}(\mathrm{C}=$ $\left.\mathrm{O}_{\text {ester }}\right), 1485 \mathrm{w}, 1455 \mathrm{w}, 1372 \mathrm{w}, 1292 \mathrm{~s}, 1256 \mathrm{~s}, 1218 \mathrm{~s}, 1184 \mathrm{vs}, 1143$ s, 1127 s, 1082 s, 1019 s, 985 m, $961 \mathrm{~m}, 790 \mathrm{~m}, 759$ s, $743 \mathrm{~s}, 714 \mathrm{~m}$ $\mathrm{cm}^{-1}$. MS: $\mathrm{m} / \mathrm{z}(\mathrm{ES}+): 246\left(\mathrm{MH}^{+}, 100 \%\right)$. This compound was too insoluble to give acceptable $\mathrm{NMR}$ spectra. $\mathrm{C}_{9} \mathrm{H}_{11} \mathrm{NO}_{5} \mathrm{~S}$ (245.25): calcd. C 44.1, H 4.5, N 5.7; found C 44.0, H 4.6, N 5.5.

(S)-2-Aminopropyl 2-Sulfobenzoate (2f): Prepared from $N$-Boc-L-alaninol $(1.05 \mathrm{~g}, 6.0 \mathrm{mmol})$, purification by recrystallisation from $\mathrm{MeCN}$ gave $2 \mathrm{f}(930 \mathrm{mg}, 60 \%)$ as a white solid. M.p. $>250{ }^{\circ} \mathrm{C}$ (dec., MeCN). $[\alpha]_{\mathrm{D}}=-22.7(c=0.3, \mathrm{EtOH}) .{ }^{1} \mathrm{H}$ NMR $(400 \mathrm{MHz}$, $\left.\mathrm{CD}_{3} \mathrm{OD}\right): \delta=8.06(\mathrm{dd}, 1 \mathrm{H}, J=7.0,1.0 ; \mathrm{Ar}), 7.65-7.59(\mathrm{~m}, 3 \mathrm{H}$, Ar), $4.68\left(\mathrm{dd}, 1 \mathrm{H}, J=12.0,3.0 ; \mathrm{OCH}_{2 \alpha}\right), 4.20(\mathrm{dd}, 1 \mathrm{H}, J=12.0$, 8.8; $\mathrm{OCH}_{2 \beta}$ ), 3.79 (ddq, $\left.1 \mathrm{H}, J=8.8,6.8,3.0 ; \mathrm{C} H \mathrm{~N}\right), 1.14$ (d, 3 $\mathrm{H}, J=6.8 ; \mathrm{Me}$ ) ppm, $\mathrm{H} / \mathrm{D}$ exchange in $\mathrm{OH}$ and $\mathrm{NH}_{2}$ groups with D from solvent. ${ }^{13} \mathrm{C}$ NMR $\left(100 \mathrm{MHz},\left[\mathrm{D}_{6}\right] \mathrm{DMSO}\right): \delta=170.2$ (CO), 140.3, 132.1, 132.0, 130.0, 129.2, 127.8, $66.9\left(\mathrm{CH}_{2}\right), 46.9$ $(\mathrm{CH}), 14.4\left(\mathrm{CH}_{3}\right) \mathrm{ppm}$. IR ( $\mathrm{KBr}$ disc $): \tilde{v}=1733 \mathrm{~s}\left(\mathrm{C}=\mathrm{O}_{\text {ester }}\right), 1626$ w, 1506 w, 1390 w, 1320 m, 1286 m, 1255 s, 1227 vs, 1211 vs, 1197 vs, 1181 vs, 1146 s, 1110 s, 1081 vs, 1024 s, 1014 s, 977 m, 951 w, 843 w, $780 \mathrm{~m}, 767 \mathrm{~s}, 739 \mathrm{~m} \mathrm{~cm}^{-1}$. MS: $\mathrm{m} / z(\mathrm{ES}+): 541\left(\mathrm{M}_{2} \mathrm{Na}^{+}\right.$, $60 \%), 519\left(\mathrm{M}_{2} \mathrm{H}^{+}, 100\right), 260\left(\mathrm{MH}^{+}, 10\right)$; found (HRMS): $\mathrm{MH}^{+}$ 260.0604, $\mathrm{C}_{10} \mathrm{H}_{14} \mathrm{NO}_{5} \mathrm{~S}$ requires 260.0593. $\mathrm{C}_{10} \mathrm{H}_{13} \mathrm{NO}_{5} \mathrm{~S}$ (259.28): calcd. C 46.3, H 5.05, N 5.4; found C 46.4, H 4.9; N 5.2.

Sodium 2-[(2-\{[(1E)-(2-Hydroxyphenyl)methylene]amino\}ethoxy)carbonyl]benzenesulfonate (6): To a stirred suspension of $\mathrm{NaH}$ $(82 \mathrm{mg}, 2.04 \mathrm{mmol})$ in anhydrous THF $(5 \mathrm{~mL})$ under an inert atmosphere was added $2 \mathrm{e}(500 \mathrm{mg} ; 2.04 \mathrm{mmol})$ at room temperature, resulting in immediate $\mathrm{H}_{2}$ evolution. The reaction was stirred for a further $20 \mathrm{~min}$ then salicylaldehyde $(0.21 \mathrm{~mL}, 2.04 \mathrm{mmol})$ was added in one portion. The reaction mixture turned yellow immediately, and was stirred for $48 \mathrm{~h}$. The reaction was quenched by the addition of $\mathrm{MeOH}(0.5 \mathrm{~mL})$, and then concentrated under vacuum to give a pale yellow solid. The crude material was taken up in hot EtOAc ( $10 \mathrm{~mL})$ and hot filtered to give $\mathbf{6}(680 \mathrm{mg}, 90 \%)$ as a pale yellow solid. M.p. $209^{\circ} \mathrm{C}$ (EtOAc). ${ }^{1} \mathrm{H}$ NMR $\left(400 \mathrm{MHz}, \mathrm{CD}_{3} \mathrm{OD}\right)$ : $\delta=8.59(\mathrm{~s}, 1 \mathrm{H}, \mathrm{NCH}), 8.02(\mathrm{dd}, 1 \mathrm{H}, J=6.1,0.8 ; \mathrm{Ar}), 7.56(\mathrm{dt}$, $1 \mathrm{H}, J=6.9,1.1$; Ar), 7.48-7.29 (m, $5 \mathrm{H}, \mathrm{Ar}), 6.89-6.86$ (m, 2 $\mathrm{H}, \mathrm{Ar}), 4.65\left(\mathrm{dt}, 2 \mathrm{H}, J=5.3,3.0 ; \mathrm{CH}_{2}\right), 4.04(\mathrm{dt}, 2 \mathrm{H}, J=5.9$, $\left.1.0 ; \mathrm{CH}_{2}\right) \mathrm{ppm}, \mathrm{OH}$ proton signal was not observed due to H/D exchange with $\mathrm{CD}_{3} \mathrm{OD} .{ }^{13} \mathrm{C}$ NMR (100 MHz, $\left.\mathrm{CD}_{3} \mathrm{OD}\right): \delta=170.7$ $\left(\mathrm{CO}_{\text {ester }}\right), 169.2\left(\mathrm{CH}_{\text {imine }}\right), 144.1(\mathrm{C}), 133.9,133.2,132.8(\mathrm{C}), 131.3$, $131.2,131.1,129.1,129.0,120.0$ (C), 119.4, 118.2, 66.1, 57.8 ppm. IR (solid state): $\tilde{v}=1739 \mathrm{~s}(\mathrm{C}=\mathrm{O}), 1632 \mathrm{~s}(\mathrm{C}=\mathrm{N}), 1500 \mathrm{w}, 1461$ w, 1286 s, 1230 s, 1197 vs, 1137 s, 1119 w, 1085 m, 1026 vs, 922 w, 757 vs, $735 \mathrm{~m} \mathrm{~cm}^{-1}$. MS: $\mathrm{m} / z(\mathrm{FAB}+)$ : $394\left(\mathrm{MNa}^{+}, 10 \%\right), 372$ $\left(\mathrm{MH}^{+}, 25\right), 329$ (15), 307 (30), 289 (15), 176 (30), 154 (100), 137 (65), 107 (20), 91 (15), 77 (15), 57 (20); found (HRMS): $\mathrm{MH}^{+}$ $372.0508, \mathrm{C}_{16} \mathrm{H}_{15} \mathrm{NO}_{6} \mathrm{SNa}$ requires 372.0518 .

Bis[2-\{(4'S)-4', 5' -dihydro-4' -isopropyloxazolyl]phenylsulfonato]palladium(II) (7): Palladium(II) acetate $(83.5 \mathrm{mg}, 0.37 \mathrm{mmol})$ and 1a (100 mg, $0.37 \mathrm{mmol})$ were refluxed at $90{ }^{\circ} \mathrm{C}$ in $\mathrm{MeCN}(7 \mathrm{~mL})$ with stirring. After $20 \mathrm{~min}$ the reaction mixture was allowed to cool to room temperature slowly, followed by cooling to $0^{\circ} \mathrm{C}$ for $12 \mathrm{~h}$, The resulting small yellow crystals were filtered to give 7 (150 mg, $100 \%$ ). M.p. $>184{ }^{\circ} \mathrm{C}$ (dec., MeCN). ${ }^{1} \mathrm{H}$ NMR $\left(400 \mathrm{MHz},\left[\mathrm{D}_{6}\right]\right.$ acetone): $\delta=8.29$ (dd, $1 \mathrm{H}, J=8.0,1.0 ; \mathrm{Ar}), 8.13-8.04(\mathrm{~m}, 3 \mathrm{H}$, $\mathrm{Ar}), 8.00(\mathrm{dt}, 1 \mathrm{H}, J=8.0,1.0 ; \mathrm{Ar}), 7.93(\mathrm{dd}, 1 \mathrm{H}, J=8.0,1.0$; Ar), $7.88(\mathrm{dt}, 1 \mathrm{H}, J=8.0,1.0$; Ar), $7.81(\mathrm{dt}, 1 \mathrm{H}, J=8.0,1.0$; Ar), 5.08 (apparent t, $\left.1 \mathrm{H}, J=9.5 \mathrm{OCH}_{2 \alpha}\right), 4.97(\mathrm{dd}, 1 \mathrm{H}, J=$ $\left.10.4,9.1 ; \mathrm{OCH}_{2 \alpha}\right), 4.92\left(\mathrm{dd}, 1 \mathrm{H}, J=9.3,6.0 ; \mathrm{OCH}_{2 \beta}\right), 4.81$ (dd, $\left.1 \mathrm{H}, J=9.1,7.5 ; \mathrm{OCH}_{2 \beta}\right), 4.37$ (ddd, $1 \mathrm{H}, J=10.4,7.5,6.0$;
$\mathrm{C} H \mathrm{~N}), 3.98(\mathrm{ddd}, 1 \mathrm{H}, J=9.9,7.5,6.0 ; \mathrm{CHN}), 2.82(\mathrm{~m}, 1 \mathrm{H}$, $\left.\mathrm{C} H \mathrm{Me}_{2}\right), 2.22(\mathrm{~m}, 1 \mathrm{H}, \mathrm{CHMe}$ ), $1.62(\mathrm{~d}, 3 \mathrm{H}, J=7.0 ; M e), 1.42$ $(\mathrm{d}, 3 \mathrm{H}, J=7.0 ; M e), 1.38$ (d, $3 \mathrm{H}, J=7.0 ; M e), 1.08(\mathrm{~d}, 3 \mathrm{H}$, $J=7.0 ; M e$ ) ppm. IR (KBr disc): $\tilde{v}=3462.6 \mathrm{~m}, 2964 \mathrm{~m}, 1624 \mathrm{vs}$ $(\mathrm{C}=\mathrm{N}), 1487 \mathrm{~m}, 1388 \mathrm{~s}, 1290 \mathrm{vs}, 1250 \mathrm{~s}, 1168 \mathrm{vs}, 1131 \mathrm{~s}, 1066 \mathrm{~m}$, 1040 w, 1022 w, 974 vs, 771 s, 746 s, $697 \mathrm{~m}, 668 \mathrm{~m}, 627 \mathrm{~m} \mathrm{~cm}^{-1}$. MS: $\quad m / z \quad\left(\mathrm{ES}^{+}\right): \quad 685 \quad\left(\mathrm{MH}^{+}(\mathrm{MeCN}), \quad 100 \%\right)$. $\mathrm{C}_{24} \mathrm{H}_{28} \mathrm{~N}_{2} \mathrm{O}_{8} \mathrm{~S}_{2} \mathrm{Pd} \cdot \mathrm{MeCN}$ (684.09): calcd. C 45.7, $\mathrm{H}$ 4.6, N 6.1; found $\mathrm{C} 45.5, \mathrm{H} 4.5, \mathrm{~N} 6.1$.

Bis[2-\{(4' $S)-4^{\prime}, 5^{\prime}$-dihydro-4' -isopropyloxazolyl]phenylsulfonato]copper(II) (8): Copper(II) acetate $(20 \mathrm{mg}, 0.1 \mathrm{mmol})$ and $\mathbf{1 a}(30 \mathrm{mg}$, $0.1 \mathrm{mmol})$ were stirred in dry $\mathrm{MeCN}(3 \mathrm{~mL})$ followed by the addition of distilled 2,6-lutidine $(12.8 \mu \mathrm{L}, 0.1 \mathrm{mmol})$. The reaction mixture was refluxed at $90{ }^{\circ} \mathrm{C}$ for $2 \mathrm{~h}$ under an inert atmosphere. The solvent was concentrated in vacuo, taken up into dichloromethane $(2 \mathrm{~mL})$ and carefully layered with light petroleum ether $(4 \mathrm{~mL})$. Large dark blue crystals formed upon standing for $24 \mathrm{~h}$. The crystals were filtered, dried under high vacuum, to give $\mathbf{8}$ (46 mg; 80\% yield). M.p. $>205{ }^{\circ} \mathrm{C}$ (dec., dichloromethane). IR (solid state): $\tilde{v}=1634 \mathrm{~m}(\mathrm{C}=\mathrm{N}), 1385 \mathrm{~m}, 1288 \mathrm{~s}, 1250 \mathrm{~m}, 1170 \mathrm{vs}$, 1142 s, 1129 s, 1068 m, 1017 w, 987 s, 929 w, 772 m, 761 m, 746 s, $698 \mathrm{~m} \mathrm{~cm}^{-1}$. MS: $\mathrm{m} / \mathrm{z}(\mathrm{ES}+)$ : $884\left(\mathrm{~L}_{3} \mathrm{Na}^{+}, 40 \%\right), 597\left(\mathrm{~L}_{2} \mathrm{Na}^{+}, 100\right)$, $288\left(\mathrm{~L}^{+}, 70\right), \mathrm{Cu}$ coordination not observed. $\mathrm{C}_{24} \mathrm{H}_{28} \mathrm{~N}_{2} \mathrm{O}_{8} \mathrm{~S}_{2} \mathrm{Cu}$ (600.17): calcd. C 48.0, H 4.7, N 4.7; found C 48.4, H 4.9, N 4.4.

Representative Preparation. 2-[(1S)-(Benzylaminomethyl)-2-methylpropylcarbamoyl]benzenesulfonic Acid (10a). Method A: To a solution of (S)-4-isopropyl-4,5-dihydrooxazol-2-yl)benzenesulfonic acid (1a) $(0.25 \mathrm{~g}, 0.93 \mathrm{mmol})$ in chlorobenzene $(45 \mathrm{~mL})$ was added benzylamine $(0.1 \mathrm{~mL}, 0.93 \mathrm{mmol})$. The reaction mixture was refluxed using a Dean-Stark trap $(10 \mathrm{~mL}$ capacity $)$ at $130^{\circ} \mathrm{C}$ for $16 \mathrm{~h}$. The reaction mixture was allowed to cool to room temperature and was concentrated under vacuum to yield a crude off-white solid. Purification by flash chromatography (dichloromethane/MeOH 5\%) gave 10a $(217 \mathrm{mg} ; 65 \%)$ as white microcrystalline solid with no further purification required; data as Method B.

Method B: To a solution of 2a ( $0.16 \mathrm{~g}, 0.54 \mathrm{mmol})$ in chlorobenzene ( $45 \mathrm{~mL})$ was added benzylamine $(59 \mu \mathrm{L}, 0.54 \mathrm{mmol})$. The reaction mixture was refluxed using a Dean-Stark trap (10 mL capacity) at $130{ }^{\circ} \mathrm{C}$ for $16 \mathrm{~h}$. The reaction mixture was allowed to cool to room temperature and was concentrated under reduced pressure to yield an off-white crude solid. Purification by flash chromatography (dichloromethane/MeOH 5\%) gave 10a (99 mg, 50\%) as a white solid. $R_{\mathrm{f}}=0.33$ (dichloromethane/MeOH $5 \%$ ). M.p. $156-158{ }^{\circ} \mathrm{C}$ (dichloromethane/MeOH). $[\alpha]_{\mathrm{D}}=-32.7\left(c=0.4, \mathrm{CHCl}_{3}\right)$. These data were attained under rigorously anhydrous conditions, normally the hemi hydrate was isolated. ${ }^{1} \mathrm{H}$ NMR $\left(400 \mathrm{MHz}, \mathrm{CDCl}_{3}\right)$ : $\delta=8.92$ (br. signal, $1 \mathrm{H}, \mathrm{SO}_{3} \mathrm{H}$ or $\mathrm{NH}_{\text {amine }}$ ), 8.60 (br. signal, $1 \mathrm{H}$, $\mathrm{SO}_{3} \mathrm{H}$ or $\left.\mathrm{NH}_{\text {amine }}\right), 8.09$ (dd, $1 \mathrm{H}, J=6.8,2.3$; $\left.\mathrm{Ar}\right), 7.75(\mathrm{~d}, 1 \mathrm{H}$, $J=9.5$; CONH), 7.56-7.47 (m, 3 H, Ar), 7.40-7.37 (m, $2 \mathrm{H}, \mathrm{Ar})$, 7.29-7.20 (m, 3 H, Ar), 4.30 (br. signal, $1 \mathrm{H}, \mathrm{CHNH}$ ), 4.17 (apparent $\mathrm{s}, 2 \mathrm{H}, \mathrm{CH}_{2} \mathrm{Ph}$ ), 3.11 (apparent $\mathrm{t}, 1 \mathrm{H}, J=12.6$; $\mathrm{CHCH}_{2 \alpha}$ ), 2.98 (dd, $1 \mathrm{H}, J=12.6,2.5 ; \mathrm{CHCH}_{2 \beta}$ ), 1.72 (oct, $1 \mathrm{H}, J=6.7 ; \mathrm{CHMe}_{2}$ ), $0.83(\mathrm{~d}, 3 \mathrm{H}, J=6.7 ; M e), 0.80(\mathrm{~d}, 3 \mathrm{H}, J=6.7 ; M e) .{ }^{1} \mathrm{H}$ NMR $\left(400 \mathrm{MHz}, \mathrm{CD}_{3} \mathrm{OD}\right): \delta=8.03(\mathrm{dd}, 1 \mathrm{H}, J=7.0,1.0 ; \mathrm{Ar})$, 7.64-7.60 (m, $4 \mathrm{H}, \mathrm{Ar}), 7.55(\mathrm{~m}, 1 \mathrm{H}, \mathrm{Ar}), 7.47-7.45(\mathrm{~m}, 3 \mathrm{H}$, Ar), 4.34-4.29 (m, $1 \mathrm{H}, \mathrm{NHCH}), 4.33\left(\mathrm{~d}, 2 \mathrm{H}, J=2.0 ; \mathrm{CH}_{2} \mathrm{Ph}\right)$, $3.37\left(\mathrm{dd}, 1 \mathrm{H}, J=11.5,2.5 ; \mathrm{CHCH}_{2 \alpha}\right), 3.21(\mathrm{dd}, 1 \mathrm{H}, J=11.5$, 12.5; $\mathrm{CHCH}_{2 \beta}$ ), 1.89 (oct, $1 \mathrm{H}, J=7.0: \mathrm{CHMe}_{2}$ ), 1.03 (d, $3 \mathrm{H}$, $J=7.0 ; M e), 1.02(\mathrm{~d}, 3 \mathrm{H}, J=7.0 ; M e) \mathrm{ppm}, \mathrm{OH}$ and $\mathrm{NH}$ proton signals not observed due to $\mathrm{H} / \mathrm{D}$ exchange with $\mathrm{CD}_{3} \mathrm{OD} .{ }^{13} \mathrm{C}$ NMR $\left(100 \mathrm{MHz}, \mathrm{CD}_{3} \mathrm{OD}\right): \delta=172.2\left(\mathrm{CO}_{a m}\right), 142.8(\mathrm{C}), 135.7(\mathrm{C}), 132.3$ (C), 132.0, 131.5, 131.4, 131.2, 130.7, 130.2, 130.1, 129.6, 128.5, 
53.2, 52.8, 51.5, $32.0(\mathrm{CH}), 19.8(\mathrm{Me}), 18.6(\mathrm{Me}) \mathrm{ppm}$. IR (solid state): $\tilde{v}=2966 \mathrm{w}, 1653 \mathrm{~s}\left(\mathrm{C}=\mathrm{O}_{a m}\right), 1594 \mathrm{w}, 1557 \mathrm{~m}, 1458 \mathrm{~m}, 1319$ w, 1241 s, 1172 vs, 1140 vs, 1081 s, 1056 w, 1033 m, 1017 vs, 830 w, 753 vs, 741 vs, 729 vs, 699 vs $\mathrm{cm}^{-1}$. MS: $\mathrm{m} / \mathrm{z}(\mathrm{FAB}+): 399$ $\left(\mathrm{MNa}^{+}, 15 \%\right), 377\left(\mathrm{MH}^{+}, 90 \%\right), 307$ (20), 154 (100), 136 (75), 120 (25), 107 (30), 91 (55), 83 (40), 69 (95), 57 (90); found (HRMS): $\mathrm{MH}^{+}$377.1519, $\mathrm{C}_{19} \mathrm{H}_{25} \mathrm{~N}_{2} \mathrm{O}_{4} \mathrm{~S}$ requires 377.1535. $\mathrm{C}_{19} \mathrm{H}_{24} \mathrm{~N}_{2} \mathrm{O}_{4} \mathrm{~S}$ (376.47): calcd. C 60.6, H 6.4, N 7.4; found C 60.2, H 6.4, N 7.1.

2-[(1S)-(Benzylaminomethyl)-3-methylbutylcarbamoyl]benzenesulfonic Acid (10b): Procedure as for 10a, method A. Purification by flash chromatography (dichloromethane/MeOH 5\%) gave 10b (164 mg, 78\%) as a viscous oil (which solidified on prolonged drying) from $1 \mathbf{b}(153 \mathrm{mg}, 0.54 \mathrm{mmol})$ and benzylamine $(59 \mu \mathrm{L}$, $0.54 \mathrm{mmol}$ ). $R_{\mathrm{f}}=0.35$ (dichloromethane $/ \mathrm{MeOH} \quad 5 \%$ ). M.p. $267-269^{\circ} \mathrm{C}$ (dichloromethane $\left./ \mathrm{MeOH} 5 \%\right)$. $[\alpha]_{\mathrm{D}}=-27.6(c=1.0$, $\left.\mathrm{CHCl}_{3}\right) .{ }^{1} \mathrm{H}$ NMR $\left(500 \mathrm{MHz}, \mathrm{CD}_{3} \mathrm{OD}\right): \delta=7.96-7.99(\mathrm{~m}, 1 \mathrm{H}$, Ar), 7.53-7.58 (m, 4 H, Ar), 7.39-7.45 (m, 5 H, Ar), 4.55 (m, 1 $\mathrm{H}, \mathrm{CHNH}), 4.28$ (apparent s, $\left.2 \mathrm{H}, \mathrm{CH}_{2} \mathrm{Ph}\right), 3.18$ (dd, $1 \mathrm{H}, J=$ 12.7, 2.9; $\left.\mathrm{CHCH}_{2 \alpha} \mathrm{NH}\right), 3.11(\mathrm{dd}, 1 \mathrm{H}, J=10.9,12.7$; $\left.\mathrm{CHCH}_{2 \beta} \mathrm{NH}\right), 1.70(\mathrm{~m}, 1 \mathrm{H}, \mathrm{CHMe}), 1.51\left(\mathrm{~m}, 1 \mathrm{H}, \mathrm{CHCH}_{2 \alpha} \mathrm{CH}\right)$, $1.28\left(\mathrm{~m}, 1 \mathrm{H}, \mathrm{CHCH}_{2 \beta} \mathrm{CH}\right), 0.98(\mathrm{~d}, 3 \mathrm{H}, J=6.7 ; \mathrm{Me}), 0.94(\mathrm{~d}, 3$ $\mathrm{H}, J=6.7 ; \mathrm{Me}) \mathrm{ppm}, \mathrm{OH}$ and $\mathrm{NH}$ proton signals not observed due to $\mathrm{H} / \mathrm{D}$ exchange with $\mathrm{CD}_{3} \mathrm{OD} .{ }^{13} \mathrm{C} \mathrm{NMR}(100 \mathrm{MHz}$, $\left.\mathrm{CD}_{3} \mathrm{OD}\right): \delta=172.2\left(\mathrm{CO}_{a m}\right), 142.8(\mathrm{C}), 135.8(\mathrm{C}), 132.2(\mathrm{C}), 131.8$ (2C), 131.4, 130.9, 130.5 (2C), 130.1, 129.1, 128.5, 53.6, 52.7, 46.2, 41.9, $26.0(\mathrm{CH}), 23.4(\mathrm{Me}), 22.0(\mathrm{Me}) \mathrm{ppm}$. IR (solid state): $\tilde{v}=$ $2957 \mathrm{w}, 1662 \mathrm{w}, 1631 \mathrm{~s}\left(\mathrm{C}=\mathrm{O}_{a m}\right), 1537 \mathrm{~m}, 1453 \mathrm{~m}, 1252 \mathrm{~s}, 1168 \mathrm{~s}$, $1142 \mathrm{~s}, 1080 \mathrm{~m}, 1017 \mathrm{~s}, 747 \mathrm{~s}, 756 \mathrm{~s}, 700 \mathrm{~s} \mathrm{~cm}^{-1}$. MS: $m / z(\mathrm{FAB}+)$ : $391\left(\mathrm{MH}^{+}, 16 \%\right), 176$ (29), 155 (25), 154 (86), 136 (70), 97 (40), 69 (76), 57 (100); found (HRMS): $\mathrm{MH}^{+} 391.1704, \mathrm{C}_{20} \mathrm{H}_{27} \mathrm{~N}_{2} \mathrm{O}_{4} \mathrm{~S}$ requires 391.1692. $\mathrm{C}_{20} \mathrm{H}_{26} \mathrm{~N}_{2} \mathrm{O}_{4} \mathrm{~S}$ (390.50): calcd. C 61.5, H 6.7, N 7.2; found $\mathrm{C} 61.4, \mathrm{H} 6.8, \mathrm{~N} 7.2$.

2-(2-Methyl-(1S)-\{[(naphthalen-2-ylmethyl)amino|methyl $\}$ propylcarbamoyl)benzenesulfonic Acid (10c): Procedure as for 10a, method A. Purification by flash chromatography (dichloromethane/MeOH $5 \%$ ) gave 10c (134 mg, 82\%) as a viscous oil from 1a $(102 \mathrm{mg}, \quad 0.38 \mathrm{mmol})$ and 1-naphthylenemethylamine $(56 \mu \mathrm{L}$, $0.38 \mathrm{mmol}$ ). $\quad R_{\mathrm{f}}=0.37$ (dichloromethane $/ \mathrm{MeOH} 5 \%$ ). M.p. $286-288{ }^{\circ} \mathrm{C}(\mathrm{MeOH}) \cdot[\alpha]_{\mathrm{D}}=-34.3\left(c=0.5, \mathrm{CHCl}_{3}\right) .{ }^{1} \mathrm{H}$ NMR $\left(400 \mathrm{MHz}, \mathrm{CD}_{3} \mathrm{OD}\right): \delta=8.26(\mathrm{dd}, 1 \mathrm{H}, J=8.5,1.0 ; \mathrm{Ar})$, 8.06-7.98 (m, $3 \mathrm{H}, \mathrm{Ar}$ ), 7.69 (dd, $1 \mathrm{H}, J=7.0,1.0$; Ar), 7.69 (dt, $1 \mathrm{H}, J=7.0,1.0$; Ar), 7.63-7.54 (m, $5 \mathrm{H}, \mathrm{Ar}), 4.89(\mathrm{~s}, 2 \mathrm{H}$, $\left.\mathrm{CH}_{2} \mathrm{Ph}\right), 4.39(\mathrm{~m}, 1 \mathrm{H}, \mathrm{C} H \mathrm{NH}), 3.47(\mathrm{dd}, 1 \mathrm{H}, J=13.0,2.5$; $\mathrm{CHCH}_{2 \alpha}$ ), 3.32 (dd, $1 \mathrm{H}, J=13.0,11.5 ; \mathrm{CHCH}_{2 \beta}$ ), 1.90 (oct, $1 \mathrm{H}$, $\left.J=7.0 ; \mathrm{C} H \mathrm{Me}_{2}\right), 1.03(\mathrm{~d}, 3 \mathrm{H}, J=7.0 ; \mathrm{Me}), 1.01(\mathrm{~d}, 3 \mathrm{H}, J=$ $7.0 ; \mathrm{Me}) \mathrm{ppm}, \mathrm{OH}$ and $\mathrm{NH}$ proton signals not observed due to $\mathrm{H} /$ D exchange with $\mathrm{CD}_{3} \mathrm{OD} .{ }^{13} \mathrm{C}$ NMR $\left(100 \mathrm{MHz}, \mathrm{CD}_{3} \mathrm{OD}\right): \delta=$ $172.2\left(\mathrm{CO}_{a m}\right), 142.7(\mathrm{C}), 135.7(\mathrm{C}), 135.3,132.8,132.0,131.4$, 131.1, 130.7, 130.0, 129.6, 128.5, 128.3, 128.1, 127.4, 126.5, 124.1, 54.8, 53.0, 32.0, $19.7\left(\mathrm{CH}_{3}\right), 18.5\left(\mathrm{CH}_{3}\right) \mathrm{ppm}$. IR (solid state): $\tilde{\mathrm{v}}=$ $2967 \mathrm{w}, 1655 \mathrm{~m}\left(\mathrm{C}=\mathrm{O}_{a m}\right), 1595 \mathrm{~m}, 1560 \mathrm{~m}, 1459 \mathrm{~m}, 1229 \mathrm{~m}, 1164$ s, $1141 \mathrm{~m}, 1082 \mathrm{~m}, 1018 \mathrm{~s}, 802 \mathrm{~m}, 799 \mathrm{~s}, 790 \mathrm{~s}, 774 \mathrm{~s}, 738 \mathrm{~s} \mathrm{~cm}^{-1}$. MS: $m / z(F A B+): 427\left(\mathrm{MH}^{+}, 5 \%\right), 391(5), 3299$ (7), 307 (40), 289 (15), 176 (10), 154 (100), 137 (65), 120 (10), 107 (20), 91 (12), 77 (15), 57 (20); found (HRMS): $\mathrm{MH}^{+} 427.1719, \mathrm{C}_{23} \mathrm{H}_{27} \mathrm{~N}_{2} \mathrm{O}_{4} \mathrm{~S}$ requires 427.1692

2-[(1S)-Benzyl-2-benzylaminoethylcarbamoyl]benzenesulfonic Acid (10d): Procedure as in 10a, method A. Purification by flash chromatography (dichloromethane/MeOH 5\%) gave 10d (125 mg, 74\%) as a white solid from $1 \mathbf{d}(127 \mathrm{mg}, 0.40 \mathrm{mmol})$ and benzylamine (59 $\mu \mathrm{L}, 0.40 \mathrm{mmol}$ ). $R_{\mathrm{f}}=0.3$ (dichloromethane $/ \mathrm{MeOH} 5 \%$ ). M.p. $107-109^{\circ} \mathrm{C}(\mathrm{MeOH}) \cdot[\alpha]_{\mathrm{D}}=-44.0\left(c=0.35, \mathrm{CHCl}_{3}\right) .{ }^{1} \mathrm{H}$ NMR
(400 MHz, $\mathrm{CDCl}_{3}$ ): $\delta=8.80$ (br. d, $2 \mathrm{H}, \mathrm{SO}_{3} \mathrm{H}$ and $\mathrm{NH}$ ), 8.06 (dd, $1 \mathrm{H}, J=6.0,1.0$; Ar), 7.65 (d, $1 \mathrm{H}, J=9.0 ; \mathrm{SO}_{2} \mathrm{NH}$ ), 7.50 (dt, 1 $\mathrm{H}, J=1.5,7.5$; Ar) overlapped by $7.46(\mathrm{dt}, 1 \mathrm{H}, J=1.4,7.4$; Ar), 7.32 (dd, $1 \mathrm{H}, J=7.4,1.4$; Ar), 7.26-7.12 (m, $7 \mathrm{H}, \mathrm{Ar}$ ), 7.04-7.02 (m, $2 \mathrm{H}, J=6.0,1.0$; Ar), 4.67 (br. signal, $1 \mathrm{H}, \mathrm{CONHCH}), 4.05$ (apparent s, $2 \mathrm{H}, \mathrm{NHCH}_{2} \mathrm{Ph}$ ), 3.07 (br. t, $1 \mathrm{H} J 11.8 ; \mathrm{CHCH}_{2 \mathrm{\alpha}} \mathrm{NH}$ ), 2.89 (br. d, $1 \mathrm{H}, J$ 11.8; $\mathrm{CHCH}_{2 \beta} \mathrm{NH}$ ), 2.76 (dd, $1 \mathrm{H}, J=13.8$, 7.9; $\mathrm{CHCH}_{2 \alpha} \mathrm{Ph}$ ), 2.70 (dd, $1 \mathrm{H}, J=13.8,7.4 ; \mathrm{CHCH}_{2 \beta} \mathrm{Ph}$ ) ppm. ${ }^{13} \mathrm{C}$ NMR (67.8 MHz, $\left.\mathrm{CD}_{3} \mathrm{OD}\right): \delta=171.9\left(\mathrm{CO}_{a m}\right), 142.8,138.3$, 135.7, 132.2, 131.8, 131.5 (2C), 131.1, 130.6 (2C), 130.3 (2C), 130.1 (2C), 129.7, 129.1, 128.5, 128.0, $52.6\left(\mathrm{CH}_{2}\right), 52.3\left(\mathrm{CH}_{2}\right), 49.6(\mathrm{CH})$, $39.2\left(\mathrm{CH}_{2}\right) \mathrm{ppm}$. The DEPT spectrum confirms the overlap of the $\mathrm{CH}$ resonance with the solvent. IR (solid state): $\tilde{v}=2359 \mathrm{w}, 1651 \mathrm{~s}$ $\left(\mathrm{C}=\mathrm{O}_{a m}\right), 1627 \mathrm{~m}, 1593 \mathrm{w}, 1546 \mathrm{~s}, 1497 \mathrm{w}, 1454 \mathrm{~m}, 1312 \mathrm{w}, 1250$ vs, $1211 \mathrm{~s}, 1162$ vs, $1138 \mathrm{~s}, 1081 \mathrm{~m}, 1018$ vs, 869 w, 753 s, 744 vs, $727 \mathrm{~m}, 699 \mathrm{vs} \mathrm{cm}{ }^{-1}$. MS: $\mathrm{m} / z(\mathrm{FAB}+)$ : $425\left(\mathrm{MH}^{+}, 30 \%\right), 307(25)$, 289 (15), 154 (100), 136 (75), 107 (25), 91 (30), 77 (25), 69 (25), 57 (35); found (HRMS): $\mathrm{MH}^{+} 425.1544, \mathrm{C}_{23} \mathrm{H}_{25} \mathrm{~N}_{2} \mathrm{O}_{4} \mathrm{~S}$ requires 425.1535. $\mathrm{C}_{23} \mathrm{H}_{24} \mathrm{~N}_{2} \mathrm{O}_{4} \mathrm{~S} \cdot 1 / 2 \mathrm{H}_{2} \mathrm{O}$ (433.52): calcd. C 63.7, $\mathrm{H} 5.8, \mathrm{~N}$ 6.5; found $\mathrm{C} 63.5, \mathrm{H} 5.55, \mathrm{~N} 6.3$.

2-\{2-Methyl-(1S)-I((1R)-phenylethylamino)methyl]propylcarbamoyl benzenesulfonic Acid (10e): Procedure as in 10a, method A. Purification by flash chromatography (dichloromethane/MeOH $5 \%)$ gave 10e $(156 \mathrm{mg}, 73 \%)$ from 1a $(148 \mathrm{mg}, 0.55 \mathrm{mmol})$ and $(1 R)$-phenylethylamine $(71 \mu \mathrm{L}, 0.55 \mathrm{mmol})$ as a viscous oil. $R_{\mathrm{f}}=$ 0.40 (dichloromethane $/ \mathrm{MeOH} \mathrm{5 \% )}$. $[\alpha]_{\mathrm{D}}=-42.0\left(c=0.5, \mathrm{CHCl}_{3}\right)$. ${ }^{1} \mathrm{H}$ NMR $\left(400 \mathrm{MHz}, \mathrm{CDCl}_{3}\right.$ ): $\delta=9.18$ (br. s, $1 \mathrm{H}, \mathrm{SO}_{3} \mathrm{H}$ or $\mathrm{NH}_{\text {amine }}$ ), 8.89 (br. s, $1 \mathrm{H}, \mathrm{SO}_{3} \mathrm{H}$ or $\left.\mathrm{NH}_{\text {amine }}\right), 8.17-8.14(\mathrm{~m}, 1 \mathrm{H}$, $\mathrm{Ar}), 8.04(\mathrm{~d}, 1 \mathrm{H}, J=9.0 ; \mathrm{CONH}), 7.72-7.70(\mathrm{~m}, 1 \mathrm{H}, \mathrm{Ar})$, 7.75-7.55 (m, $4 \mathrm{H}, \mathrm{Ar}), 7.44-7.39$ (m, $3 \mathrm{H}, \mathrm{Ar}$ ), 4.62 (br. q, $J$ 6.5, $1 \mathrm{H}, \mathrm{NHCHPh}, 4.43$ (br. s, $1 \mathrm{H}, \mathrm{NHCH} i \mathrm{Pr}$ ), 3.13 (br. t, $J 12.4,1$ $\mathrm{H}, \mathrm{CHCH}_{2 \alpha}$ ), 2.86 (br. d, $\left.J 12.4,1 \mathrm{H}, \mathrm{CHCH}_{2 \beta}\right), 1.73$ (d, $3 \mathrm{H}, J=$ 6.8; $\mathrm{CH}(\mathrm{Ph}) \mathrm{Me}] 1.66$ (oct, $1 \mathrm{H}, J=6.8 ; \mathrm{CHMe}$ ), $0.82(\mathrm{~d}, 3 \mathrm{H}$, $J=6.8$; Me), 0.77 (d, $3 \mathrm{H}, J=6.8$; Me) ppm. ${ }^{13} \mathrm{C}$ NMR $(100 \mathrm{MHz}$, $\left.\mathrm{CDCl}_{3}\right): \delta=169.5\left(\mathrm{CO}_{a m}\right), 141.3(\mathrm{C}), 135.6(\mathrm{C}), 133.9(\mathrm{C}), 130.9$, 130.4, 129.8, 129.6, 129.5, 127.9, 127.5, 57.8, 50.4, 46.9, 31.0, 20.7, $19.1(\mathrm{Me}), 18.2(\mathrm{Me}) \mathrm{ppm}$. IR (solid state): $\tilde{v}=2969 \mathrm{w}, 1652 \mathrm{~m}$ $\left(\mathrm{C}=\mathrm{O}_{a m}\right), 1594 \mathrm{w}, 1556 \mathrm{~m}, 1456 \mathrm{~m}, 1318 \mathrm{w}, 1241 \mathrm{~s}, 1174 \mathrm{vs}, 1140$ s, 1080 s, 1016 vs, 782 w, 762 s, 735 s, 702 vs cm$^{-1}$. MS: $m / z$ (ES+): $781\left(\mathrm{M}_{2} \mathrm{H}^{+}, 100 \%\right), 391\left(\mathrm{MH}^{+}, 50\right)$; found (HRMS): $\mathrm{MH}^{+}$ 391.1656, $\mathrm{C}_{20} \mathrm{H}_{27} \mathrm{~N}_{2} \mathrm{SO}_{4}$ requires 391.1691.

2-\{3-Methyl-(1S)-[((1R)-1-phenylethylamino)methyl]butylcarbamoyl $\}$ benzenesulfonic Acid (10f): Procedure as in 10a, method A. Purification by flash chromatography (dichloromethane/MeOH $5 \%)$ gave $\mathbf{1 0 f}(189 \mathrm{mg}, 75 \%)$ as a viscous oil from $\mathbf{1 b}(0.81 \mathrm{~g}$, $2.85 \mathrm{mmol})$ and $(1 R)$-phenylethylamine $(367 \mu \mathrm{L}, 2.85 \mathrm{mmol}) . R_{\mathrm{f}}=$ 0.40 (dichloromethane/MeOH 5\%). $[\alpha]_{\mathrm{D}}=-40.0\left(c=0.1, \mathrm{CHCl}_{3}\right)$. ${ }^{1} \mathrm{H}$ NMR $\left(400 \mathrm{MHz}, \mathrm{CDCl}_{3}\right): \delta=9.23$ (br. s, $1 \mathrm{H}, \mathrm{SO}_{3} \mathrm{H}$ or $\mathrm{NH}_{\text {amine }}$ ), 8.91 (br. s, $1 \mathrm{H}, \mathrm{SO}_{3} \mathrm{H}$ or $\left.\mathrm{NH}_{\text {amine }}\right), 8.14$ (dd, $1 \mathrm{H}, J=$ 7.0, 1.5; Ar), 7.89 (d, $1 \mathrm{H}, J=9.0 ; \mathrm{CONH}), 7.67$ (dd, $1 \mathrm{H}, J=$ 7.0, 1.5; Ar), 7.58-7.53 (m, 4 H, Ar), 7.43-7.41 (m, 3 H, Ar), 4.66 (br. q, $J$ 7, $1 \mathrm{H}, \mathrm{CH}(\mathrm{Ph}) \mathrm{Me}$; overlapped by (br. s, $1 \mathrm{H}, \mathrm{CONHCH}$ ), $3.08\left(\mathrm{t}, 1 \mathrm{H}, J=12.0 ; \mathrm{CHCH}_{2 \alpha} \mathrm{NH}\right), 2.79(\mathrm{~d}, 1 \mathrm{H}, J=12.0$; $\left.\mathrm{CHCH}_{2 \beta} \mathrm{NH}\right), 1.75(\mathrm{~d}, 3 \mathrm{H}, J=7.0 ; \mathrm{CH}(\mathrm{Ph}) \mathrm{Me}$ ); $1.57-1.48(\mathrm{~m}$, $\left.1 \mathrm{H}, \mathrm{CH} \mathrm{Me}_{2}\right), 1.44-1.34\left(\mathrm{~m}, 1 \mathrm{H}, \mathrm{CHCH}_{2 \alpha} \mathrm{CH}\right), 1.10(\mathrm{~m}, 1 \mathrm{H}$, $\left.\mathrm{CHCH}_{2 \beta} \mathrm{CH}\right), 0.87$ (d, $\left.3 \mathrm{H}, J=6.9 ; M e\right), 0.80$ (d, $3 \mathrm{H}, J=6.9$; $\mathrm{Me}) \mathrm{ppm} .{ }^{13} \mathrm{C}$ NMR $\left(100 \mathrm{MHz}, \mathrm{CDCl}_{3}\right): \delta=169.3\left(\mathrm{CO}_{a m}\right), 141.3$ (C), 135.7 (C), 134.0 (C), 130.8, 130.2, 130.1, 129.6, 129.5, 127.8, 127.5, 58.2, 49.1, 44.0, 41.9, 24.8, $22.7\left(\mathrm{CH}_{3}\right), 22.0\left(\mathrm{CH}_{3}\right), 20.7$ $\left(\mathrm{CH}_{3}\right) \mathrm{ppm}$. IR (solid state): $\tilde{\mathrm{v}}=2959 \mathrm{w}, 1653 \mathrm{~m}\left(\mathrm{CO}_{a m}\right), 1595 \mathrm{w}$, 1548 m, 1457 m, 1387 w, 1305 w, 1235 s, 1174 vs, 1140 s, 1082 s, 1017 vs, 850 w, 758 vs, 743 s, 702 vs, $692 \mathrm{~s} \mathrm{~cm}^{-1}$. MS: $m / z$ (FAB+): 
$427\left(\mathrm{MNa}^{+}, 55 \%\right), 405\left(\mathrm{MH}^{+}, 100\right), 307(25), 169(25), 154(95)$, 136 (75), 120 (20), 105 (55), 89 (20), 77 (25), 69 (20), 57 (35), 55 (30); found (HRMS): $\mathrm{MH}^{+}$405.1852, $\mathrm{C}_{21} \mathrm{H}_{29} \mathrm{~N}_{2} \mathrm{O}_{4} \mathrm{~S}$ requires 405.1848. $\mathrm{C}_{21} \mathrm{H}_{28} \mathrm{~N}_{2} \mathrm{O}_{4} \mathrm{~S} \cdot \mathrm{H}_{2} \mathrm{O}$ (422.54): calcd. C 59.7, H 7.2, N 6.6; found $\mathrm{C} 60.15, \mathrm{H} 6.8, \mathrm{~N} 6.8$.

X-ray Crystallographic Studies: Crystals of 1a and 2a, were grown from $\mathrm{EtOH}$, those of $\mathbf{7}$ and $\mathbf{8}$ from $\mathrm{MeCN}$, that of $\mathbf{1 0 c}$ from $\left[\mathrm{D}_{4}\right] \mathrm{MeOH}$. X-ray diffraction data were collected on SMART ${ }^{[15]}$ area detector diffractometers (APEX and SMART1000), using graphite-monochromated Mo- $K_{\alpha}$ radiation $(\lambda=0.71073 \AA)$. SAINT ${ }^{[15]}$ software was used to integrate the data sets and apply the Lorentz and polarisation corrections. An absorption and incident beam decay correction was performed using SADABS for complexes 7 and $\mathbf{8}^{[16]}$ No absorption or decay correction was made for species 1a, 2a and 10c. The structures were solved by direct methods using SHELXS-97. ${ }^{[17]}$ The structures were refined on $F^{2}$ using full-matrix least-squares (SHELXL-97). ${ }^{[18]}$ Unless otherwise stated, all fully occupied non-hydrogen atoms were refined with anisotropic displacement parameters. Hydrogen atoms were placed in geometrically calculated positions except those of the $\mathrm{MeCN}$ molecules in 7 and the $\mathrm{NH}_{3}$ and methyl hydrogens in compounds 2a and 1a, which were all located by difference Fourier synthesis.
Geometrically refined hydrogen atoms were refined as part of a riding model, with the hydrogen atoms assigned isotropic displacement parameters 1.2 times the parent atom $U_{\text {eq }}$, except the methyl atoms where it was 1.5 times. Suitable geometric constraints were applied to all disorder models. In both $\mathbf{8}$ and 10c a solvent region could not be satisfactorily modelled in terms of atomic sites and the electron density in this region was calculated and accounted for using the SQUEEZE procedure. ${ }^{[19]}$ Neutral atom scattering factors and anomalous dispersion corrections were taken from the International Tables for Crystallography. ${ }^{[20]}$ Crystallographic data are summarised in Table 1.

CCDC-246699 to -246703 contain the supplementary crystallographic data for this paper. These data can be obtained free of charge at www.ccdc.cam.ac.uk/conts/retrieving.html [or from the Cambridge Crystallographic Data Centre, 12 Union Road, Cambridge CB2 1EZ, UK; Fax: +44-1223-336-033; E-mail: deposit@ccdc.cam.ac.uk].

\section{Acknowledgments}

We acknowledge generous support of the European Commission through project FP6-505267-1 (LIGBANK) and the COST-D24

Table 1. Crystal structure data of compounds 1a, 2a, 7, 8, 10c

\begin{tabular}{|c|c|c|c|c|c|}
\hline & 1a & $2 a$ & 7 & 8 & $10 \mathrm{c}$ \\
\hline formula & $\mathrm{C}_{12} \mathrm{H}_{15} \mathrm{NO}_{4} \mathrm{~S}$ & $\mathrm{C}_{12} \mathrm{H}_{17} \mathrm{NO}_{5} \mathrm{~S}$ & $\mathrm{C}_{26} \mathrm{H}_{31} \mathrm{~N}_{3} \mathrm{O}_{8} \mathrm{PdS}_{2}$ & $\mathrm{C}_{26} \mathrm{H}_{32} \mathrm{Cl}_{4} \mathrm{CuN}_{2} \mathrm{O}_{8} \mathrm{~S}_{2}$ & $\mathrm{C}_{24} \mathrm{H}_{29} \mathrm{~N}_{2} \mathrm{O}_{5} \mathrm{~S}$ \\
\hline$M_{\mathrm{w}}$ & 269.31 & 287.33 & 684.06 & 770.00 & 457.55 \\
\hline crystal system & orthorhombic & monoclinic & monoclinic & hexagonal & orthorhombic \\
\hline space group & $P 2_{1} 2_{1} 2_{1}$ & $P 2_{1}$ & $P 2_{1}$ & $P 6_{1}$ & $P 2_{1} 2_{1} 2_{1}$ \\
\hline$a(\AA)$ & $8.1800(9)$ & $8.7089(12)$ & $8.1006(5)$ & $19.2656(14)$ & $10.520(3)$ \\
\hline$b(\AA)$ & $10.575(1)$ & $12.666(2)$ & $16.6538(10)$ & - & $12.047(4)$ \\
\hline$c(\AA)$ & $14.683(2)$ & $12.559(2)$ & $10.9452(7)$ & $15.1909(16)$ & $20.267(6)$ \\
\hline$\alpha\left(^{\circ}\right)$ & 90 & 90 & 90 & 90 & 90 \\
\hline$\beta\left({ }^{\circ}\right)$ & 90 & $107.374(2)$ & $104.939(1)$ & 90 & 90 \\
\hline$\gamma\left({ }^{\circ}\right)$ & 90 & 90 & 90 & 120 & 90 \\
\hline$V\left(\AA^{3}\right)$ & $1270.2(4)$ & $1322.2(6)$ & $1426.79(3)$ & $4882.9(7)$ & $2568(2)$ \\
\hline $\mathrm{Z}$ & 4 & 4 & 2 & 6 & 4 \\
\hline$\rho_{\text {calcd. }}\left[\mathrm{g} \mathrm{cm}^{-3}\right]$ & 1.408 & 1.443 & 1.592 & 1.571 & 1.183 \\
\hline absorption coefficient $\left[\mathrm{mm}^{-1}\right]$ & 0.261 & 0.26 & 0.85 & 1.18 & 0.16 \\
\hline$F(000)$ & 568 & 608 & 700 & 2370 & 972 \\
\hline crystal size (mm) & $0.31 \times 0.20 \times 0.18$ & $0.52 \times 0.24 \times 0.12$ & $0.54 \times 0.32 \times 0.24$ & $0.40 \times 0.12 \times 0.10$ & $0.58 \times 0.30 \times 0.19$ \\
\hline \multicolumn{6}{|l|}{ Data collection } \\
\hline$T[\mathrm{~K}]$ & $150(2)$ & $150(2)$ & $150(2)$ & $150(2)$ & $150(2)$ \\
\hline$\theta_{\min }-\theta_{\max }\left({ }^{\circ}\right)$ & $2.4-28.8$ & $2.3-28.8$ & $1.9-28.6$ & $2.4-28.9$ & $2.2-26.4$ \\
\hline scan type & $\omega$ & $\omega$ & $\omega$ & $\omega$ & $\omega$ \\
\hline$h, k, l$ ranges & $\begin{array}{l}-10 \text { to } 7,-10 \text { to } 14, \\
-19 \text { to } 12\end{array}$ & $\begin{array}{l}-11 \text { to } 11,-17 \text { to } 14 \\
-7 \text { to } 16\end{array}$ & $\begin{array}{l}-10 \text { to } 10,-21 \text { to } 22, \\
-14 \text { to } 12\end{array}$ & $\begin{array}{l}-25 \text { to } 7,-21 \text { to } 22, \\
-20 \text { to } 19\end{array}$ & $\begin{array}{l}-13 \text { to } 13,-15 \text { to } 14, \\
-25 \text { to } 25\end{array}$ \\
\hline total reflections collected & 8010 & 7361 & 11281 & 14314 & 19909 \\
\hline independent reflections & $2803\left(R_{\mathrm{int}}=0.0635\right)$ & $5306\left(R_{\mathrm{int}}=0.038\right)$ & $6231\left(R_{\mathrm{int}}=0.014\right)$ & $7458\left(R_{\mathrm{int}}=0.052\right)$ & $5264\left(R_{\mathrm{int}}=0.039\right)$ \\
\hline reflections with $I>2 \sigma(I)$ & 2327 & 4500 & 6149 & 4693 & 4802 \\
\hline absorption correction & none & none & $\begin{array}{l}T_{\min .}=0.653 \\
T_{\max .}=0.801\end{array}$ & $\begin{array}{l}T_{\min .}=0.716 \\
T_{\max .}=1.000\end{array}$ & none \\
\hline \multicolumn{6}{|l|}{ Refinement } \\
\hline final $R$ indices $[I>2 \sigma(I)]$ & $\begin{array}{l}R_{1}=0.064 \\
w R_{2}=0.156\end{array}$ & $\begin{array}{l}R_{1}=0.036 \\
w R_{2}=0.082\end{array}$ & $\begin{array}{l}R_{1}=0.018 \\
w R_{2}=0.047\end{array}$ & $\begin{array}{l}R_{1}=0.051 \\
w R_{2}=0.091\end{array}$ & $\begin{array}{l}R_{1}=0.057 \\
w R_{2}=0.150\end{array}$ \\
\hline final $R$ indices (all data) & $\begin{array}{l}R_{1}=0.0751 \\
w R_{2}=0.01624\end{array}$ & $\begin{array}{l}R_{1}=0.0443 \\
w R_{2}=0.0852\end{array}$ & $\begin{array}{l}R_{1}=0.0179 \\
w R_{2}=0.0471\end{array}$ & $\begin{array}{l}R_{1}=0.089 \\
w R_{2}=0.100\end{array}$ & $\begin{array}{l}R_{1}=0.061 \\
w R_{2}=0.152\end{array}$ \\
\hline goodness-of-fit on $F^{2}$ & 1.01 & 0.96 & 1.05 & 1.02 & 1.11 \\
\hline absolute structure parameter & $0.03(13)$ & $-0.03(7)$ & $0.005(12)$ & $0.020(15)$ & $0.03(11)$ \\
\hline final $(\Delta / \sigma)_{\max }$ & 0.001 & 0.001 & 0.001 & 0.001 & 0.001 \\
\hline largest diff. peak and hole $\left(\mathrm{e} \cdot \AA^{-3}\right)$ & $0.86 /-0.97$ & $0.33 /-0.43$ & $0.35 /-0.29$ & $0.33 /-0.41$ & $0.29 /-0.29$ \\
\hline
\end{tabular}


programme. One of us (RIR) is grateful to the EPSRC for the award of a studentship.

${ }^{[1]}$ Overviews of library preparations: ${ }^{[1 a]}$ Handbook of Combinatorial Chemistry (Eds.: K. C. Nicolaou, R. Hanko, W. Hartwig), vol. 1-2, Wiley-VCH, Weinheim, 2002. ${ }^{[1 \mathrm{~b}]} \mathrm{C}$. Gennari, U. Piarulli, Chem. Rev. 2003, 103, 3071-3100. ${ }^{[1 c]}$ A. Lee, J. G. Breitenbucher, Current Opinion in Drug Discovery and Development 2003, 6, 494-508.

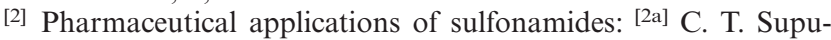
ran, A. Innocenti, A. Mastrolorenzo, A. Scozzafava, Mini-Reviews in Medicinal Chemistry 2004, 4, 189-200. ${ }^{[2 b]}$ C. T. Supuran, A. Casini, A. Scozzafava, Med. Res. Rev. 2003, 23, 535-558. ${ }^{[2 c]}$ A. Scozzafava, T. Owa, A. Mastrolorenzo, C. T. Supuran, Cur. Med. Chem. 2003, 10, 925-953.

[3] Sulfonamides in selective catalysis: [3a] F. Lake, C. Moberg, Russ. J. Org. Chem. 2003, 39, 436-452. ${ }^{[3 b]}$ P. J. Walsh, Acc. Chem. Res. 2003, 36, 739-749. [3c] J. Zhang, H.-B. Zhou, R.G. Xie, Cur. Org. Chem. 2002, 6, 865-890.

[4] [4a] H. C. Kolb, K. B. Sharpless, Drug Discovery Today 2003, 8, 1128-1137. ${ }^{[4 b]}$ H. C. Kolb, M. G. Finn, K. B. Sharpless, Angew. Chem. Int. Ed. 2001, 40, 2004-2021; H. C. Kolb, M G. Finn, K. B. Sharpless, Angew. Chem. 2001, 113, 2056-2075.

[5] [5a] H. Moehrke, H. Koch, H. von Freyberg, German Patent 1953, DE 865305 (Farberke Hoechst AG, Meister Lucius and Bruning) [Chem. Abstr. 1958, 52, 113808]. ${ }^{[5 b]}$ J. R. Ashby, E. M. Ramage, J. Heterocycl. Chem. 1978, 15, 1501-1502. [5c] A D. Wolf, M. P. Rorer, Eur. Pat. Appl. 1983, EP 83975 A2 19830720 (du Pont de Nemours) [Chem. Abstr. 1983, 99, 175812].

[6] For example, 2-phenyl-4,5-dihydrooxazole in the presence of concentrated aqueous $\mathrm{HCl}$ reportedly undergoes ring opening to form $N$-(2-chloroethyl)benzamide. See: R. Gabriel, M. Heymann, Ber. Dtsch. Chem. Ges. 1890, 23, 2502; B. Goldberg, F. Kelly, J. Chem. Soc. 1948, 1919-1925.
[7] Inspection of the CSD database (www.ccdc.cam.ac.uk) reveals the structures BEYZIO, BEYZIO01/02, OTANAC and RATBOD to be of relevance.

[8] A. A. D. Tulloch, S. Winston, A. A. Danopoulos, G. Eastham, M. B. Hursthouse, Dalton Trans. 2003, 699-708.

[9] For example, structures ADUREW, WABXUS and XABPEN from the CSD database (www.ccdc.cam.ac.uk) show $\mathrm{Cu}-\mathrm{O}$ distances in the range 2.386-2.471, and $\mathrm{Cu}-\mathrm{O}-\mathrm{S}$ angles covering $126.33-173.04^{\circ}$.

${ }^{[10]}$ Alternative preparations relying on activation of $\mathrm{ArSO}_{3} \mathrm{H}$ groups through $\mathrm{SOCl}_{2},(\mathrm{COCl})_{2} / \mathrm{DMF}$ or $\mathrm{POCl}_{3}$ were not successful in our case due to the presence of the wrong tautomeric form of the oxazolinesulfonic acid $\mathbf{1}$.

${ }^{[11]}$ R. I. Robinson, S. Woodward, unpublished work, see supplementary data for this paper $\left({ }^{13} \mathrm{C}\right.$ NMR spectra for compounds 1, 2, 6-11).

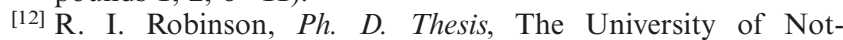
tingham, 2003.

${ }^{[13]}$ D. A. Quagliato, P. M. Andrae, E. M. Matelan, J. Org. Chem. 2000, 65, 5037-5042.

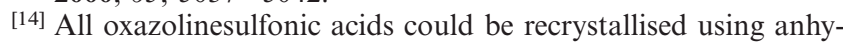
drous ethanol, apart from 2-((S)-4-benzyl-4,5-dihydrooxazol-2yl)benzenesulfonic acid (1d).

${ }^{[15]}$ SMART and SAINT Area-Detector Control and Integration Software, Bruker AXS, Madison, 1997.

${ }^{[16]}$ G. M. Sheldrick, SADABS: Program for Scaling and Correction of Area Detector Data, University of Göttingen, 1996.

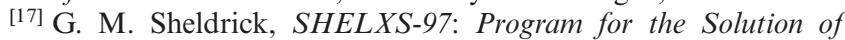
Crystal Structures, University of Göttingen, 1997.

${ }^{[18]}$ G. M. Sheldrick, SHELXL-97: Program for the Refinement of Crystal Structures, University of Göttingen, 1997.

${ }^{[19]}$ P. v.d. Sluis, A. L. Spek, Acta Crystallogr., Sect. A 1990, 46, 194-201.

${ }^{[20]}$ International Tables for Crystallography, vol. C (Ed.: A. J. C. Wilson), Kluwer Academic, Dordrecht, 1992.

Received August 6, 2004 